\title{
HARMONIC ANALYSIS AND BMO-SPACES OF FREE ARAKI-WOODS FACTORS
}

\author{
MARTIJN CASPERS
}

\begin{abstract}
We consider semi-group BMO-spaces associated with arbitrary von Neumann algebras and prove interpolation theorems. This extends results by Junge-Mei for the tracial case. We give examples of multipliers on free Araki-Woods algebras and in particular we find $L_{\infty} \rightarrow$ BMO multipliers. We also provide $L_{p}$-bounds for a natural generalization of the Hilbert transform.
\end{abstract}

\section{INTRODUCTION}

Recall that the BMO-norm of a classical integrable function $f: \mathbb{R}^{n} \rightarrow \mathbb{C}$ is defined as

$$
\|f\|_{\mathrm{BMO}}=\sup _{Q \in \mathcal{Q}} \frac{1}{|Q|} \int_{Q}\left|f(s)-\oint_{Q} f\right|^{2} d s,
$$

where $\oint_{Q} f$ is the average of $f$ over $Q$ and $\mathcal{Q}$ is the set of all cubes in $\mathbb{R}^{n}$. The importance of the BMO-norm and BMO-spaces lies in the fact that they arise as end-point estimates/spaces for the bounds of linear maps on function spaces on $\mathbb{R}^{n}$. This includes many singular integral operators, Calderón-Zygmund operators and Fourier multipliers. BMO-spaces are by FeffermanStein duality [FeSt72] dual to Hardy spaces and provide optimal bounds for the Hilbert transform. By interpolation BMO-spaces form an effective tool to obtain $L_{p}$-bounds of multipliers.

BMO-spaces can also be studied through semi-groups. Consider for example the heat semigroup $\mathcal{S}:=\left(\Phi_{t}\right)_{t \geq 0}:=\left(e^{-t \Delta}\right)_{t \geq 0}$ with Laplacian $\Delta$ acting on $L_{\infty}\left(\mathbb{R}^{n}\right)$. Then alternatively the BMO-norm may be realized through an equivalent (semi-)norm

$$
\|f\|_{\mathrm{bmo}_{\mathcal{S}}}=\sup _{t \geq 0}\left\|\left|\Phi_{t}(f)\right|^{2}-\Phi_{t}\left(|f|^{2}\right)\right\|^{\frac{1}{2}} .
$$

BMO-spaces associated with more general semi-groups were first studied in [StVa74], [Var85] and much more recently in [XuYa05a], [XuYa05b]. See also [Gra08], [Gra09]. These concern semi-groups on measure spaces, which from our viewpoint is the commutative situation.

The development and exploration of structural properties of $\mathrm{C}^{*}$-algebras and von Neumannn algebras led to the demand of a thorough development of harmonic analysis on non-commutative spaces. After the founding work by Eymard defining the Fourier algebra of a group [Eym64], the study of its $L_{\infty}$-multipliers turned out to have tremendous impact on the structure of operator algebras (see e.g. [BrOz08]). In recent years also the $L_{p}$-theory was pursued. Under suitable Hörmander-Mikhlin type conditions several multiplier theorems were established for group von Neumann algebras [JMP14], [CPPR15], [GJP17a] and vector valued harmonic analysis [Cad17], [Par09]. On quantum spaces several surprising multiplier theorems have been achieved [CXY13],

Date: February 14, 2018.

2010 Mathematics Subject Classification. Primary: 47A20, 47A57, 47D07.

Key words and phrases. BMO-spaces, Markov semi-groups, complex interpolation, non-commutative $L_{p}$-spaces, free Gaussians, Fourier multipliers. 
[Ric16]. See also [XXX16], [GJP17b]. These results naturally raise questions about end-point estimates and optimal bounds for multipliers.

Parallel to this development semi-groups on non-commutative measure spaces have played a more and more important role in recent years. They lead to strong applications in noncommutative potential theory and quantum probability, see e.g. [CiSa03], [CFK14]. Semi-groups naturally appear in approximation properties of von Neumann algebras [JoMa04], [CaSk15]. Also the approach by Ozawa-Popa [OzPo10] and Peterson [Pet09] yields new deformation-rigidity properties of von Neumann algebras through the theory of semi-groups and derivations (see also [Avs11]).

In [JuMe12] Junge and Mei pursued the theory of non-commutative semi-group BMO-spaces associated with non-commutative measure spaces. They introduce several notions of BMO starting from a Markov semi-group on a tracial von Neumann algebra. Relations between these spaces are studied and interpolation results are obtained. A crucial ingredient of their approach is formed by Markov dilations of semi-groups that allows one to 'intertwine' semi-group BMO-spaces with BMO-spaces associated with martingales and derive results from this probabilistic martingale setting.

The first aim of this paper is the study of BMO-spaces associated with an arbitrary $\sigma$-finite von Neumann algebra. We take the natural definition using a faithful normal state which is not necessarily tracial anymore as a starting point. We extend interpolation results from [JuMe12, Theorem 5.2] to the arbitrary setting under a modularity assumption on the Markov semi-group. The modularity assumption is necessary to carry out our proof through Haagerup's reduction method and due to the fact that the probabilistic martingale BMO-spaces in [JuPe14] are studied (in principle only) in the tracial setting. This culminates in Theorem 3.15, which briefly states the following. Let $\mathcal{S}$ be a modular Markov semi-group admitting a reversed Markov dilation with a.u. continuous path on a $\sigma$-finite von Neumann algebra $\mathcal{M}$. We have

$$
\left[\operatorname{bmo}_{\mathcal{S}}^{\circ}(\mathcal{M}), L_{p}^{\circ}(\mathcal{M})\right]_{1 / q} \approx_{p q} L_{p q}^{\circ}(\mathcal{M}) .
$$

Other interpolation theorems for Poisson semi-groups and different BMO-spaces are then discussed in Section 4. Proofs here are similar and some aspects in fact simplify.

In Section 5 we give examples of multiplier theorems of non-tracial von Neumann algebras, namely free Araki-Woods factors (see [Shl97]). The first part of Section 5 introduces a natural generalization of the (free) Hilbert transform. We get $L_{p}$-bounds through Cotlar's trick. Recently in [MeRi16] Mei and Ricard obtained the analogous result for free group factors. We also give examples of $L_{\infty} \rightarrow$ BMO multipliers and show that the interpolation result of (1.1) applies. We leave it as an open question whether the Hilbert transform admits a $L_{\infty} \rightarrow$ BMO-estimate (or even a $\mathrm{BMO} \rightarrow$ BMO-estimate as for the classical Hilbert transform [FeSt72], [Gra09]). In Section 5.3 we construct a reversed Markov dilation for the semi-groups that we use on free ArakiWoods factors. The construction is essentially due to Ricard [Ric08] which is combined with an ultraproduct argument to go from the discrete to continuous case.

\section{Preliminaries AND NOtation}

We start with some general conventions. For general operator theory we refer to [Tak02] and for operator spaces to [EfRu00], [Pis02]. Throughout the paper $\mathcal{M}$ will be a von Neumann algebra with fixed normal faithful state $\varphi . \mathcal{S}=\left(\Phi_{t}\right)_{t \geq 0}$ will be a fixed Markov semi-group, see Section 2.3 for details. $\left(\sigma_{s}^{\varphi}\right)_{s \in \mathbb{R}}$ denotes the modular automorphism group of $\varphi$, see [Tak03] for modular theory. 
2.1. General notation. For the complex interpolation method we refer to the book [BeLö76]. See also [Cas13] for a short summary and the relation to non-commutative $L_{p}$-spaces. Let $S$ be the strip of all complex numbers with imaginary part in the interval $[0,1]$. For a compatible couple of Banach spaces $(X, Y)$ denote $\mathcal{F}(X, Y)$ for the space of functions $S \rightarrow X+Y$ that (i) are continuous on $S$ and analytic on the interior of $S$, (ii) $f(s) \in X$ and $f(i+s) \in Y$, (iii) $\|f(s)\|_{X} \rightarrow 0$ and $\|f(i+s)\|_{Y} \rightarrow 0$ as $|s| \rightarrow \infty$. We write $(X, Y)_{\theta}$ for the interpolation space at parameter $\theta \in[0,1]$.

2.2. $L_{p}$-spaces associated with an arbitrary von Neumann algebra. This paper establishes results on interpolation and harmonic analysis on non-tracial von Neumann algebras. The $L_{p^{-}}$-spaces of such von Neumann algebras can be described through constructions introduced by Haagerup [Haa77], [Ter81] and Connes-Hilsum [Con80], [Hil81] (the latter in fact relies on Haagerup's construction to treat sums and products of unbounded operators). In principle we use the definition of Hilsum [Hil81], though it is easy to recast each of the statements in terms of [Haa77].

For a general von Neumann algebra $\mathcal{M}$ we let $\phi^{\prime}$ be a fixed normal, semi-finite, faithful weight on the commutant $\mathcal{M}^{\prime}$. For a normal, semi-finite weight $\varphi$ on $\mathcal{M}$ we write $D_{\varphi}$ for Connes's spatial derivative $d \varphi / d \phi^{\prime}$ [Con80], [Ter81]. For every von Neumann algebra in this paper $\phi^{\prime}$ is implicitly fixed; it can be chosen arbitrary and $\phi^{\prime}$ will be suppressed in the notation. $L_{p}(\mathcal{M})$ with $\mathcal{M} \subseteq B(\mathcal{H})$ is defined as all closed densely defined operators $x$ on $\mathcal{H}$ such that $|x|^{p}=D_{\varphi}$ for some $\varphi \in \mathcal{M}_{*}^{+}$. Then $\|x\|_{p}=\|\varphi\|^{1 / p}$. Products and sums of elements in (different) $L_{p^{-} \text {-spaces }}$ are understood as strong products and strong sums (so closure of the product and sum). We will omit these closures in the notation. $L_{p}$-spaces satisfy classical properties as Hölder estimates. In particular for all $x \in \mathcal{M}$ and $\varphi \in \mathcal{M}_{*}$ positive we have $D_{\varphi}^{\frac{1}{2 p}} x D_{\varphi}^{\frac{1}{2 p}} \in L_{p}(\mathcal{M})$. In fact such elements are (norm) dense in $L_{p}(\mathcal{M})$ for $1 \leq p<\infty$.

We turn $L_{p}(\mathcal{M}), 1 \leq p \leq \infty$ into a compatible couple (or compatible scale) of Banach spaces. Assume $\mathcal{M}$ is $\sigma$-finite, meaning that there exists a faithful, normal state $\varphi$ on $\mathcal{M}$. Then there is a contractive embedding $\kappa_{p}^{\varphi}: L_{p}(\mathcal{M}) \rightarrow L_{1}(\mathcal{M})$ determined by

$$
D_{\varphi}^{\frac{1}{2 p}} x D_{\varphi}^{\frac{1}{2 p}} \mapsto D_{\varphi}^{\frac{1}{2}} x D_{\varphi}^{\frac{1}{2}}
$$

Considering $L_{p}(\mathcal{M})$ as (non-isometric) linear subspaces of $L_{1}(\mathcal{M})$ we may and will interpret intersections, sum spaces and interpolation spaces of $L_{p}(\mathcal{M})$ and $L_{r}(\mathcal{M})$ within $L_{1}(\mathcal{M})$. Such spaces depend on $\varphi$ and we will usually mark $\varphi$ in the notation (we shall need a transition between the tracial and non-tracial case). For example $\left[L_{p}(\mathcal{M}), L_{r}(\mathcal{M})\right]_{\theta}^{\varphi}$ will denote the complex interpolation spaces between $L_{p}(\mathcal{M})$ and $L_{r}(\mathcal{M})$ at parameter $\theta \in[0,1]$ with respect to the embeddings of $L_{p}(\mathcal{M})$ and $L_{r}(\mathcal{M})$ in $L_{1}(\mathcal{M})$ through $\kappa_{p}^{\varphi}$ and $\kappa_{r}^{\varphi}$.

2.3. Semi-groups. We recall preliminaries on semi-groups.

Definition 2.1. A map $\Phi: \mathcal{M} \rightarrow \mathcal{M}$ is called Markov if it is normal ucp (unital completely positive) and $\varphi \circ \Phi=\varphi$ (where $\varphi$ is the fixed faithful normal state on $\mathcal{M}$ ). Through complex interpolation between $\mathcal{M}$ and $L_{1}(\mathcal{M})$, a Markov map has a contractive $L_{2}$-implementation given by

$$
\Phi^{(2)}: D_{\varphi}^{\frac{1}{4}} x D_{\varphi}^{\frac{1}{4}} \rightarrow D_{\varphi}^{\frac{1}{4}} \Phi(x) D_{\varphi}^{\frac{1}{4}} .
$$

A Markov map is called KMS-symmetric if $\Phi^{(2)}$ is self-adjoint. A Markov map is called GNSsymmetric if $\varphi\left(\Phi(x)^{*} y\right)=\varphi\left(x^{*} \Phi(y)\right)$ for all $x, y \in \mathcal{M}$. $\Phi$ is called $\varphi$-modular if for every $s \in \mathbb{R}$ we have $\Phi \circ \sigma_{s}^{\varphi}=\sigma_{s}^{\varphi} \circ \Phi$. 
If $\Phi$ is $\varphi$-modular then it is KMS-symmetric if and only if it is GNS-symmetric.

Definition 2.2. A family $\left(\Phi_{t}\right)_{t>0}$ is called a semi-group if $\Phi_{s+t}=\Phi_{s} \circ \Phi_{t}$ and for every $x \in \mathcal{M}$ we have $\Phi_{t}(x) \rightarrow x$ in the strong topology as $t \searrow 0$. A semi-group $\left(\Phi_{t}\right)_{t>0}$ is called Markov, KMSsymmetric or $\varphi$-modular if for each $t \geq 0$ the map $\Phi_{t}$ is respectively Markov, KMS-symmetric or $\varphi$-modular.

By interpolation between $L_{1}$ and $L_{\infty}$ we may in fact define $\Phi_{t}^{(p)}$ as (the closure of)

$$
\Phi_{t}^{(p)}: L_{p}(\mathcal{M}) \rightarrow L_{p}(\mathcal{M}): D_{\varphi}^{\frac{1}{2 p}} x D_{\varphi}^{\frac{1}{2 p}} \mapsto D_{\varphi}^{\frac{1}{2 p}} \Phi_{t}(x) D_{\varphi}^{\frac{1}{2 p}},
$$

see [JuXu07, Lemma 7.1]. If $\Phi_{t}$ is $\varphi$-modular then for $x$ analytic,

$$
\begin{aligned}
& \Phi_{t}^{(2)}\left(x D_{\varphi}^{\frac{1}{2}}\right)=\Phi_{t}^{(2)}\left(D_{\varphi}^{\frac{1}{4}} \sigma_{i / 4}^{\varphi}(x) D_{\varphi}^{\frac{1}{4}}\right)=D_{\varphi}^{\frac{1}{4}} \Phi_{t}\left(\sigma_{i / 4}^{\varphi}(x)\right) D_{\varphi}^{\frac{1}{4}} \\
= & D_{\varphi}^{\frac{1}{4}} \sigma_{i / 4}^{\varphi}\left(\Phi_{t}(x)\right) D_{\varphi}^{\frac{1}{4}}=\Phi_{t}(x) D_{\varphi}^{\frac{1}{2}} .
\end{aligned}
$$

For $1 \leq p<\infty$ let $A_{p} \geq 0$ be the unbounded generator of our Markov semi-group, which may be characterized by

$$
\operatorname{Dom}\left(A_{p}\right)=\left\{\xi \in L_{p}(\mathcal{M}) \mid \lim _{t \searrow 0} t^{-1}\left(\Phi_{t}^{(p)}(\xi)-\xi\right) \text { exists }\right\}
$$

and for $\xi \in \operatorname{Dom}\left(A_{p}\right), A_{p} \xi=\lim _{t \searrow 0} t^{-1}\left(\xi-\Phi_{t}^{(p)}(\xi)\right)$. We have $\exp \left(-t A_{p}\right)=\Phi_{t}^{(p)}$. We also set,

$$
L_{p}^{\circ}(\mathcal{M})=\left\{\xi \in L_{p}(\mathcal{M}) \mid \lim _{t \rightarrow \infty} \Phi_{t}^{(p)}(\xi)=0\right\} .
$$

Note that as $\varphi$ is a normal faithful state, we have an inclusion

$$
\kappa_{r, p}^{\varphi}:=\left(\kappa_{r}^{\varphi}\right)^{-1} \circ \kappa_{p}^{\varphi}: L^{p}(\mathcal{M}) \subseteq L^{r}(\mathcal{M}): D_{\varphi}^{\frac{1}{2 p}} x D_{\varphi}^{\frac{1}{2 p}} \mapsto D_{\varphi}^{\frac{1}{2 r}} x D_{\varphi}^{\frac{1}{2 r}}, \quad x \in \mathcal{M},
$$

whenever $r \leq p$ and this inclusion is a contractive mapping that intertwines $\Phi_{t}^{(p)}$ and $\Phi_{t}^{(r)}$. It follows therefore that $\operatorname{Dom}\left(A_{p}\right) \subseteq \operatorname{Dom}\left(A_{r}\right)$. We also set,

$$
\mathcal{M}^{\circ}=\left\{x \in \mathcal{M} \mid \Phi_{t}(x) \rightarrow 0 \quad \sigma \text {-weakly }\right\} .
$$

And for notational convenience $L_{\infty}^{\circ}(\mathcal{M})=\mathcal{M}^{\circ}$.

Lemma 2.3. For $1 \leq r \leq p \leq \infty$ we have $L_{p}^{\circ}(\mathcal{M}) \subseteq L_{r}^{\circ}(\mathcal{M})$ for the inclusion (2.3).

Proof. Assume $p \neq \infty$. Take $y \in L_{p}^{\circ}(\mathcal{M})$ then $\Phi_{t}^{(p)}(y) \rightarrow 0$. So $\Phi_{t}^{(r)}\left(\kappa_{r, p}^{\varphi}(y)\right)=\kappa_{r, p}^{\varphi}\left(\Phi_{t}^{(p)}(y)\right) \rightarrow 0$ which is equivalent to $\kappa_{r, p}^{\varphi}(y) \in L_{r}^{\circ}(\mathcal{M})$. Assume $p=\infty$. Take $y \in \mathcal{M}^{\circ}$ so that $\Phi_{t}^{(p)}(y) \rightarrow 0$ strongly. Then $\Phi_{t}^{(p)}\left(\kappa_{p, \infty}^{\varphi}(y)\right)=D_{\varphi}^{\frac{1}{2 p}} \Phi_{t}(y) D_{\varphi}^{\frac{1}{2 p}} \rightarrow 0$ by [JuSh05, Lemma 1.3].

Remark 2.4. Suppose that the state $\varphi$ is almost periodic, meaning that its modular operator $\nabla_{\varphi}$ has a complete set of eigenspaces. In this case there is the following averaging trick in order to assure the existence of $\varphi$-modular semi-groups (see e.g. [OkTo15, Theorem 4.15] for a similar argument). By [Con73, Lemma 3.7.3] there exists a compact group $\widehat{\Gamma}$ with group homomorphism $\rho: \mathbb{R} \rightarrow \widehat{\Gamma}$ with dense range and a continuous unitary representation $s \mapsto U_{s}, s \in \widehat{\Gamma}$ on $B\left(L_{2}(\mathcal{M})\right)$ such that for $t \in \mathbb{R}$ we get $\nabla_{\varphi}^{i t}=U_{\rho(t)}$. Let $\Phi$ be a Markov map on $\mathcal{M}$. Then the map

$$
\Phi^{a v}=\int_{\widehat{\Gamma}} \operatorname{ad}\left(U_{s}^{*}\right) \circ \Phi \circ \operatorname{ad}\left(U_{s}\right) d s
$$


is also Markov. Moreover, it is $\varphi$-modular as

$$
\begin{aligned}
& \Phi^{a v} \circ \sigma_{t}^{\varphi}=\Phi^{a v} \circ \operatorname{ad}\left(\nabla_{\varphi}^{i t}\right)=\int_{\widehat{\Gamma}} \operatorname{ad}\left(U_{s}^{*}\right) \circ \Phi \circ \operatorname{ad}\left(U_{s+\rho(t)}\right) d s \\
= & \int_{\widehat{\Gamma}} \operatorname{ad}\left(U_{s}^{*} U_{\rho(t)}\right) \circ \Phi \circ \operatorname{ad}\left(U_{s}\right) d s=\sigma_{t}^{\varphi} \circ \Phi^{a v} .
\end{aligned}
$$

Similarly, if $\left(\Phi_{t}\right)_{t \geq 0}$ is a Markov semi-group then $\left(\Phi_{t}^{a v}\right)_{t \geq 0}$ is a Markov semi-group that is moreover $\varphi$-modular.

2.4. Markov dilations of semi-groups. The following terminology was introduced in [JuMe12] (see also [Ana06] and [Ric08]). It forms the crucial condition that is being used in Junge and Mei their proofs of interpolation results.

Definition 2.5. A standard Markov dilation of a semi-group $\mathcal{S}=\left(\Phi_{t}\right)_{t \geq 0}$ on a von Neumann algebra $\mathcal{M}$ with normal faithful state $\varphi$ consists of: (1) A von Neumann algebra $\mathcal{N}$ with faithful normal state $\varphi_{\mathcal{N}},(2)$ an increasing filtration $\left(\mathcal{N}_{s}\right)_{s \geq 0}$ with $\varphi_{\mathcal{N}}$-preserving conditional expectations $\mathcal{E}_{s}: \mathcal{N} \rightarrow \mathcal{N}_{s},(3)$ state preserving $*$-homomorphisms $\pi_{s}: \mathcal{M} \rightarrow \mathcal{N}_{s}$ such that

$$
\mathcal{E}_{s}\left(\pi_{t}(x)\right)=\pi_{s}\left(\Phi_{t-s}(x)\right), \quad s<t, x \in \mathcal{M} .
$$

Definition 2.6. A reversed Markov dilation of a semi-group $\mathcal{S}=\left(\Phi_{t}\right)_{t \geq 0}$ on a von Neumann algebra $\mathcal{M}$ with normal faithful state $\varphi$ consists of: (1) A von Neumann algebra $\mathcal{N}$ with faithful normal state $\varphi_{\mathcal{N}},(2)$ a decreasing filtration $\left(\mathcal{N}_{s}\right)_{s \geq 0}$ with $\varphi_{\mathcal{N}}$-preserving conditional expectations $\mathcal{E}_{s}: \mathcal{N} \rightarrow \mathcal{N}_{s},(3)$ state preserving $*$-homomorphisms $\pi_{s}: \mathcal{M} \rightarrow \mathcal{N}_{s}$ such that

$$
\mathcal{E}_{s}\left(\pi_{t}(x)\right)=\pi_{s}\left(\Phi_{s-t}(x)\right), \quad t<s, x \in \mathcal{M} .
$$

We call a (standard or reversed) Markov dilation modular if in their definitions we have moreover

$$
\sigma_{t}^{\varphi \mathcal{N}} \circ \pi_{s}=\pi_{s} \circ \sigma_{t}^{\varphi}, s \geq 0, t \in \mathbb{R} .
$$

Without loss of generality for a standard Markov dilation we may assume that $\mathcal{N}_{s}$ is generated by $\pi_{t}(x), t \leq s, x \in \mathcal{M}$ and $\mathcal{N}=\left(\cup_{s \geq 0} \mathcal{N}_{s}\right)^{\prime \prime}$. Then the condition (2.6) implies that $\sigma^{\varphi \mathcal{N}}$ preserves $\mathcal{N}_{s}$ for every $s \geq 0$.

We typically denote standard/reversed Markov dilations by means of a triple $\left(\mathcal{N}_{t}, \pi_{t}, \mathcal{E}_{t}\right)_{t \geq 0}$. The von Neumann algebra $\mathcal{N}$ is then implicitly understood as the $\sigma$-weak closure of $\cup_{t \geq 0} \mathcal{N}_{t}$.

Definition 2.7. An $L_{\infty}$-martingale $\left(x_{t}\right)_{t \geq 0}$ in a von Neumann algebra $\mathcal{N}$ with faithful normal state $\psi$ and with filtration $\left(\mathcal{N}_{t}\right)_{t \geq 0}$ has a.u. continuous path if for every $T>0, \epsilon>0$ there exists a projection $e \in \mathcal{N}$ with $\psi(1-e)<\epsilon$ such that $[0, T] \rightarrow \mathcal{N}: t \mapsto x_{t} e$ is continuous.

We require Lemma 2.8 which was already observed in [JuMe12, p. 716] and [JuMe12, p. 637]. For properties of vector valued $L_{p}$-spaces we refer to [Pis96]. Let $x=\left(x_{t}\right)_{t \geq 0}$ be a martingale as in Definition 2.7. Let $2<p<\infty$. Let $\sigma=\left\{t_{1}, \ldots, t_{n_{\sigma}}\right\}$ be a (finite) set of elements $0<t_{1}<$ $\ldots<t_{n_{\sigma}}<\infty$. We write

$$
\|x\|_{h_{p}^{d}(\sigma)}=\left(\sum_{t_{i} \in \sigma}\left\|x_{t_{i+1}}-x_{t_{i}}\right\|_{L_{p}}^{p}\right)^{\frac{1}{p}},
$$

and then $\|x\|_{h_{p}^{d}}=\lim _{\sigma, \mathcal{U}}\|x\|_{h_{p}^{d}(\sigma)}$ for any ultrafilter containing the filter base of tails. This yields a norm, which is independent of the choice of ultrafilter [JuPe14]. Note that the $h_{p}^{d}(\sigma)$-norm is just the $L_{p}\left(\ell_{p}(\sigma)\right)$-norm [Pis96] of the martingale difference sequence $d_{i}(x)=x_{t_{i+1}}-x_{t_{i}}$. It follows 
straight from the definitions that if $\mathcal{Q}$ is a von Neumann subalgebra of $\mathcal{N}$ with expectation $\mathcal{E}_{\mathcal{Q}}$ satisfying for all $t \geq 0, \mathcal{E}_{\mathcal{Q}} \circ \mathcal{E}_{t}=\mathcal{E}_{t} \circ \mathcal{E}_{\mathcal{Q}}$. Then for every martingale $x=\left(x_{t}\right)_{t \geq 0}$ in $\mathcal{N}$ we get

$$
\left\|\mathcal{E}_{\mathcal{Q}}(x)\right\|_{h_{p}^{d}} \leq\|x\|_{h_{p}^{d}} .
$$

Lemma 2.8. If a martingale $x=\left(x_{t}\right)_{t \geq 0}$ has a.u. continuous path then $\|x\|_{h_{p}^{d}}=0$ for all $p>2$.

Proof. We use the notation of Definition 2.7. By Doob's inequality [Jun02] for every $2<p<\infty$ and $T>0$ there exists a continuous function $f:[0, T] \rightarrow \mathcal{N}$ and an element $a \in L_{p}(\mathcal{N})$ such that $x_{t}=f(t) a$. Then taking the ultralimit over all finite subsets $\sigma \subseteq[0, T]$ we get $\|x\|_{L_{p}\left(\ell_{\infty}^{c}(\sigma)\right)} \rightarrow 0$. By interpolation

$$
\|x\|_{h_{p}^{d}(\sigma)} \leq\left\|d_{j}(x)\right\|_{L_{p}\left(\ell_{\infty}^{c}(\sigma)\right)}^{\theta}\left\|d_{j}(x)\right\|_{L_{p}\left(\ell_{2}^{c}(\sigma)\right.}^{1-\theta},
$$

with $\theta=p / 2$. Let $\sigma=\left\{t_{1}<\ldots<t_{n}\right\}$ be a finite subset of $[0, T]$. Set $d_{j}(x)=x_{t_{j+1}}-x_{t_{j}}$. The norm $\left\|\left(d_{j}(x)\right)_{j}\right\|_{L_{p}\left(\ell_{2}^{c}(\sigma)\right.}$ can be upper estimated by the norm $\|x\|_{p}$ by the Burkholder-Gundy inequality [HJX10, Theorem 6.4] and in particular is uniformly bounded in $\sigma$. Then as we already showed that $\left\|d_{j}(x)\right\|_{L_{p}\left(\ell_{\infty}^{c}(\sigma)\right)} \rightarrow 0$ we conclude.

Because modular Markov dilations are state preserving homomorphisms, they extend to maps

$$
\pi_{s}^{(p)}: L_{p}(\mathcal{M}) \rightarrow L_{p}\left(\mathcal{N}_{s}\right): D_{\varphi}^{\frac{1}{2 p}} x D_{\varphi}^{\frac{1}{2 p}} \rightarrow D_{\varphi_{\mathcal{N}}}^{\frac{1}{2 p}} \pi_{s}(x) D_{\varphi_{\mathcal{N}}}^{\frac{1}{2 p}}, \quad x \in \mathcal{M}, s \geq 0 .
$$

These are $\mathcal{M}-\mathcal{M}$ bimodule maps in the sense that $\pi_{s}(x) \pi_{s}^{(p)}(y) \pi_{s}(z)=\pi_{s}^{(p)}(x y z)$ for $x, z \in \mathcal{M}$ and $y \in L_{p}(\mathcal{M})$.

We shall need a notion of almost uniform continuity of Markov dilations. These notions were considered in [JuMe12] (see also [JuMe10]) and play an important role for embeddings of various BMO-spaces. Our notion differs from what is used in [JuMe12, p. 725], which assumes a.u. continuity of two martingales $m(f)$ and $n(f)$. But actually the proof of the interpolation result in the first statement of [JuMe12, Theorem 5.2 (ii)] only uses a.u. continuity of the martingale $m(f)$, which is what we need (the second statement of [JuMe12, Theorem 5.2 (ii)] requires more).

Definition 2.9. A reversed Markov dilation $\left(\mathcal{N}_{t}, \pi_{t}, \mathcal{E}_{t}\right)_{t \geq 0}$ for a Markov semi-group $\mathcal{S}=\left(\Phi_{t}\right)_{t \geq 0}$ on a von Neumann algebra $\mathcal{M}$ has a.u. continuous path if there exists a $\sigma$-weakly dense subset $B \subseteq \mathcal{M}$ such that for all $x \in B$ the $L_{\infty}$-martingale

$$
m(x)=\left(m_{t}(x)\right)_{t \geq 0}=\left(\pi_{t} \circ \Phi_{t}(x)\right)_{t \geq 0} .
$$

has a.u. continuous path.

Remark 2.10. In the work in progress [JRS] it is proved that Markov semi-groups on finite von Neumann algebras always admit a standard (as well as reversed) Markov dilation with a.u. continuous path.

\section{Semi-group BMO FOR $\sigma$-Finite von Neumann ALgebras}

In this section we generalize some of the interpolation results from [JuMe12], in particular Theorem 3.8, for finite von Neumann algebras to arbitrary $\sigma$-finite von Neumann algebras.

Throughout this section we let $\mathcal{S}=\left(\Phi_{t}\right)_{t \geq 0}$ be a Markov semi-group on a $\sigma$-finite von Neumann algebra $\mathcal{M}$ with fixed normal faithful state $\varphi$. In order to do reduction we must assume later that $\mathcal{S}$ is $\varphi$-modular. Furthermore in order to interpret BMO-spaces (see Section 3.3) as interpolation spaces we must assume that $\mathcal{S}$ is GNS-symmetric (which in case the semi-group is $\varphi$-modular is equivalent to being KMS-symmetric). 
3.1. The Haagerup reduction method. Let $G=\cup_{n \in \mathbb{N}} \frac{1}{n} \mathbb{Z}$ equipped with the discrete topology. We set $\mathcal{R}=\mathcal{M} \rtimes_{\sigma^{\varphi}} \mathrm{G}$ which is the subalgebra of $\mathcal{M} \otimes \mathcal{B}\left(\ell_{2}(\mathrm{G})\right)$ generated by operators

$$
l_{g}=1 \otimes \lambda_{g}, \quad \pi_{\varphi}(x)=\sum_{g \in \mathrm{G}} \sigma_{-g}^{\varphi}(x) \otimes e_{g, g}, \quad g \in \mathrm{G}, x \in \mathcal{M} .
$$

The map $\pi_{\varphi}$ identifies $\mathcal{M}$ as a subalgebra of $\mathcal{R}$ and hence we often omit it. For every $\gamma \in \widehat{\mathrm{G}}$ there exists an automorphism $\theta_{\gamma}: \mathcal{R} \rightarrow \mathcal{R}$ called the dual action that is determined by $\theta_{\gamma}\left(\pi_{\varphi}(x)\right)=$ $\pi_{\varphi}(x), \theta_{\gamma}\left(l_{g}\right)=\langle\gamma, g\rangle_{\widehat{\mathrm{G}}, \mathrm{G}} l_{g}$ with $x \in \mathcal{M}, g \in \mathrm{G}$. There exists a normal conditional expectation $\mathcal{E}_{\mathcal{M}}: \mathcal{R} \rightarrow \pi_{\varphi}(\mathcal{M}) \simeq \mathcal{M}$ that is given by

$$
\mathcal{E}_{\mathcal{M}}(x)=\int_{\gamma \in \widehat{\mathrm{G}}} \theta_{\gamma}(x) d \gamma, \quad x \in \mathcal{R}
$$

We set $\widetilde{\varphi}=\varphi \circ \pi_{\varphi}^{-1} \circ \mathcal{E}_{\mathcal{M}}$, which is a normal faithful state on $\mathcal{R}$ that restricts to $\varphi$ on $\mathcal{M}$. We define $b_{n}=-i \log \left(\lambda_{2^{-n}}\right)$ where we use the principal branch of the logarithm so that $0 \leq \Im(\log (z))<2 \pi$. Then set $a_{n}=2^{n} b_{n}, h_{n}=e^{-a_{n}}$ and

$$
\widetilde{\varphi}_{n}=h_{n}^{\frac{1}{2}} \widetilde{\varphi_{n}} h_{n}^{\frac{1}{2}}, \quad \mathcal{R}_{n}:=\mathcal{R}_{\widetilde{\varphi}_{n}},
$$

Here $\mathcal{R}_{\widetilde{\varphi}_{n}}:=\left\{x \in \mathcal{R} \mid \sigma_{t}^{\widetilde{\varphi}_{n}}(x)=x\right\}$ is the centralizer of $\widetilde{\varphi}_{n}$. By construction the operator $h_{n}$ is boundedly invertible. Furthermore,

$$
D_{\widetilde{\varphi}}^{i t} h_{n} D_{\widetilde{\varphi}}^{-i t}=h_{n}, \quad \text { and } \quad D_{\widetilde{\varphi}_{n}}^{i t} h_{n} D_{\widetilde{\varphi}_{n}}^{-i t}=h_{n} .
$$

Now we recall the following theorem from [HJX10] (see also [CPPR15, Section 7] for the weight case), which is known as the reduction method.

Theorem 3.1. With the above notation we have:

(1) Each $\mathcal{R}_{n}$ is finite with normal faithful trace $\widetilde{\varphi}_{n}$.

(2) There exist normal conditional expectations $\mathcal{E}_{n}: \mathcal{R} \rightarrow \mathcal{R}_{n}$ such that $\widetilde{\varphi} \circ \mathcal{E}_{n}=\widetilde{\varphi}$ and $\sigma_{t}^{\widetilde{\varphi}} \circ \mathcal{E}_{n}=\mathcal{E}_{n} \circ \sigma_{t}^{\widetilde{\varphi}}$ for all $t \in \mathbb{R}$.

(3) For each $x \in \mathcal{R}$ we have $\mathcal{E}_{n}(x) \rightarrow x$ in the $\sigma$-strong topology.

The following lemma is standard. We included a sketch of the proof for convenience of the reader.

Lemma 3.2. Let $\Phi$ be a $\varphi$-modular Markov map on $\mathcal{M}$. Then there exists a unique normal $\widetilde{\varphi}$-modular extension $\widetilde{\Phi}$ on $\mathcal{R}$ such that

$$
\widetilde{\Phi}\left(\pi_{\varphi}(x) \lambda_{g}\right)=\pi_{\varphi}(\Phi(x)) \lambda_{g}, \quad x \in \mathcal{R}, g \in \widehat{\mathrm{G}} .
$$

In particular we have

$$
\widetilde{\Phi}\left(h_{n}^{i t} \pi_{\varphi}(x) h_{n}^{-i t}\right)=h_{n}^{i t} \widetilde{\Phi}\left(\pi_{\varphi}(x)\right) h_{n}^{-i t}, x \in \mathcal{M} .
$$

Moreover if $\Phi$ is Markov then so is $\widetilde{\Phi}$ and if $\left(\Phi_{t}\right)_{t \geq 0}$ is a Markov semi-group then so is $\left(\widetilde{\Phi}_{t}\right)_{t \geq 0}$ for both $\widetilde{\varphi}$ and $\widetilde{\varphi}_{n}$. If $\left(\Phi_{t}\right)_{t \geq 0}$ is KMS-symmetric, then so is $\left(\widetilde{\Phi}_{t}\right)_{t \geq 0}$ for both $\widetilde{\varphi}$ and $\widetilde{\varphi}_{n}$.

Proof. As $\mathcal{R}=\mathcal{M} \rtimes_{\sigma^{\varphi}} \mathrm{G} \subseteq \mathcal{M} \otimes \mathcal{B}\left(\ell_{2}(\mathrm{G})\right)$. We let $\widetilde{\Phi}$ be the restriction of $\Phi \otimes \operatorname{id}_{\mathcal{B}\left(\ell_{2}(\mathrm{G})\right)}$ to $\mathcal{R}$. Using that $\Phi$ commutes with the modular group of $\varphi$ (3.4) follows. If $\Phi$ is Markov then for $x \in \mathcal{M}, g \in \mathrm{G}$,

$$
\begin{aligned}
& \widetilde{\varphi} \circ \widetilde{\Phi}\left(\pi_{\varphi}(x) \lambda_{g}\right)=\widetilde{\varphi}\left(\pi_{\varphi}(\Phi(x)) \lambda_{g}\right)=\varphi \circ \mathcal{E}_{\mathcal{M}}\left(\pi_{\varphi}(\Phi(x)) \lambda_{g}\right) \\
= & \varphi(\Phi(x)) \delta_{g, 0}=\varphi(x) \delta_{g, 0}=\widetilde{\varphi}\left(\pi_{\varphi}(x) \lambda_{g}\right) .
\end{aligned}
$$


So $\widetilde{\Phi}$ is Markov. As $h_{n}$ is contained in $1 \otimes \mathcal{L}(\mathrm{G})$ we have that $\widetilde{\Phi}\left(h_{n}^{*} h_{n}\right)=h_{n}^{*} h_{n}=\widetilde{\Phi}\left(h_{n}\right)^{*} \widetilde{\Phi}\left(h_{n}\right)$. So $h_{n}$ is in the multiplicative domain of $\widetilde{\Phi}$ [BrOz08, Proposition 1.5.6] and so $\widetilde{\Phi}\left(h_{n}^{\frac{1}{2}} y h_{n}^{\frac{1}{2}}\right)=h_{n}^{\frac{1}{2}} \widetilde{\Phi}(y) h_{n}^{\frac{1}{2}}$. It follows that $\widetilde{\Phi}$ is Markov for $\widetilde{\varphi}_{n}$. Also $\widetilde{\Phi}\left(h_{n}^{-i t} h_{n}^{i t}\right)=1=h_{n}^{-i t} h_{n}^{i t}=\widetilde{\Phi}\left(h_{n}^{-i t}\right) \widetilde{\Phi}\left(h_{n}^{i t}\right)$, so that also $h_{n}^{i t}$ is in the multiplicative domain of $\widetilde{\Phi}$ and so (3.5) follows from [BrOz08, Proposition 1.5.6].

Furthermore, since $\Phi_{t}$ is strongly continuous $\widetilde{\Phi}_{t}$ is strongly continuous. As the modular group $\sigma^{\widetilde{\varphi}}$ is determined by $\sigma_{t}^{\widetilde{\varphi}}\left(\pi_{\varphi}(x)\right)=\pi_{\varphi}\left(\sigma_{t}^{\varphi}(x)\right), x \in \mathcal{M}$ and $\sigma_{t}^{\widetilde{\varphi}}\left(l_{g}\right)=l_{g}, g \in \mathrm{G}$ it follows that $\widetilde{\Phi}_{t} \circ \sigma_{s}^{\widetilde{\varphi}}=\sigma_{s}^{\widetilde{\varphi}} \circ \widetilde{\Phi}_{t}$. From the definition one finds that $\widetilde{\varphi}\left(\widetilde{\Phi}_{t}(x) y\right)=\widetilde{\varphi}\left(x \widetilde{\Phi}_{t}(y)\right)$ which for $\widetilde{\varphi}$-modular semi-groups yields that the semi-group is KMS-symmetric (see (2.2)).

From this point let $\widetilde{\mathcal{S}}=\left(\widetilde{\Phi}_{t}\right)_{t \geq 0}$ be the extension of the Markov semi-group of Theorem 3.2 of a prefixed $\varphi$-modular Markov semi-group $\mathcal{S}=\left(\Phi_{t}\right)_{t \geq 0}$.

3.2. Reducing Markov dilations. In this section we show that Markov dilations and a.u. continuity behaves well with reduction.

Proposition 3.3. Suppose that $\mathcal{S}$ is $\varphi$-modular and admits a standard (resp. reversed) $\varphi$-modular Markov dilation. Then the semi-group $\widetilde{\mathcal{S}}$ admits a standard (resp. reversed) $\widetilde{\varphi}$-modular Markov dilation. Moreover, if the reversed Markov dilation of $\mathcal{S}$ has a.u. continuous path, then the reversed Markov dilation of $\widetilde{\mathcal{S}}$ may be chosen to have a.u. continuous path.

Proof. As before write $\mathcal{S}=\left(\Phi_{t}\right)_{t \geq 0}$ for the semi-group and $\widetilde{\mathcal{S}}=\left(\widetilde{\Phi}_{t}\right)_{t \geq 0}$ for the crossed product extension as in Lemma 3.2.

Part 1: Dilations. Let $\left(\mathcal{N}_{s}, \pi_{s}, \mathcal{E}_{s}\right)_{s \geq 0}$ be a $\varphi$-modular reversed Markov dilation for $\mathcal{S}$ with respect to a normal faithful state $\psi$ on $\mathcal{N}$. Let $\mathcal{O}=\mathcal{N} \rtimes_{\sigma^{\psi_{\mathcal{N}}}} \mathrm{G}$ and $\mathcal{O}_{s}=\mathcal{N}_{s} \rtimes_{\sigma^{\psi} \mathcal{N}_{\mathcal{N}}} \mathrm{G}$ and equip it with the dual weight $\widetilde{\psi}=\psi \circ \pi_{\psi}^{-1} \circ \int_{\widehat{\mathrm{G}}} \theta_{\gamma} d \gamma$. Because $\sigma_{t}^{\psi} \circ \pi_{s}=\pi_{s} \circ \sigma_{t}^{\varphi}$ it follows that $\pi_{s}$ extends uniquely to a normal map $\tilde{\pi}_{s}: \mathcal{R} \rightarrow \mathcal{O}$ that intertwines the modular groups of $\sigma^{\psi}$ and $\sigma^{\varphi}$. Similarly because for $\varphi_{\mathcal{N}}$-preserving conditional expectations we have $\mathcal{E}_{s} \circ \sigma_{t}^{\psi}=\sigma_{t}^{\psi} \circ \mathcal{E}_{s}, s \geq 0, t \in \mathbb{R}$ we get conditional expectations $\widetilde{\mathcal{E}}_{s}: \mathcal{O} \rightarrow \mathcal{O}_{s}$. In particular $\left(\mathcal{O}_{s}\right)_{s \geq 0}$ is filtered as the operators $\pi_{\psi}(x), x \in \cup_{s \geq 0} \mathcal{N}_{s}, l_{t}, t \in \mathrm{G}$ are dense in $\mathcal{O}$. We claim that $\left(\mathcal{O}_{s}, \widetilde{\pi}_{s}, \widetilde{\mathcal{E}}_{s}\right)_{s \geq 0}$ is a reversed Markov dilation.

For $g \in \mathrm{G}$ let $l_{g}^{\mathcal{R}} \in \mathcal{R}$ and $l_{g}^{\mathcal{O}} \in \mathcal{O}$ be the operators $l_{g}$ of (3.1) in these respective von Neumann algebras. It follows from the relations $\widetilde{\Phi}_{t} \circ \pi_{\varphi}=\pi_{\varphi} \circ \Phi_{t}, \widetilde{\pi}_{s} \circ \pi_{\varphi}=\pi_{\psi} \circ \pi_{s}$ and $\pi_{\psi} \circ \mathcal{E}_{s}=\widetilde{\mathcal{E}}_{s} \circ \pi_{\psi}$ that for $x \in \mathcal{M}, t<s$,

$$
\begin{aligned}
& \widetilde{\pi}_{s} \circ \widetilde{\Phi}_{s-t}\left(\pi_{\varphi}(x) l_{g}^{\mathcal{R}}\right)=\widetilde{\pi}_{s} \circ \pi_{\varphi}\left(\Phi_{s-t}(x)\right) l_{g}^{\mathcal{O}}=\pi_{\psi} \circ \pi_{s} \circ \Phi_{s-t}(x) l_{g}^{\mathcal{O}} \\
= & \pi_{\psi} \circ \mathcal{E}_{s} \circ \pi_{t}(x) l_{g}^{\mathcal{O}}=\widetilde{\mathcal{E}}_{s} \circ \widetilde{\pi}_{t}\left(\pi_{\varphi}(x) l_{g}^{\mathcal{R}}\right) .
\end{aligned}
$$

Therefore (2.4) follows by density.

This proves the first statement for reversed Markov dilations, for standard Markov dilations the proof is similar.

Part 2: A.u. continuity of the paths. Suppose now that the reversed Markov dilation $\left(\mathcal{N}_{s}, \pi_{s}, \mathcal{E}_{s}\right)_{s \geq 0}$ in part 1 has a.u. continuous path. By Definition 2.9 there exists a $\sigma$-weakly dense subspace $B \subseteq \mathcal{M}$ such that for $x \in B, T>0, \epsilon>0$ there is a projection $e \in \mathcal{N}$ with $\psi(1-e)<\epsilon$ such that the map $[0, T] \ni t \mapsto m_{t}(x) e$ is continuous with $m_{t}(x):=\pi_{t}\left(\Phi_{t}(x)\right)$. Let $\widetilde{e}=\pi_{\psi}(e)$. Then we 
have for $g \in \mathrm{G}$ that

$$
\begin{aligned}
& \widetilde{\pi}_{t}\left(\widetilde{\Phi}_{t}\left(l_{g} \pi_{\varphi}(x)\right)\right) \widetilde{e}=l_{g} \widetilde{\pi}_{t}\left(\widetilde{\Phi}_{t}\left(\pi_{\varphi}(x)\right)\right) \pi_{\psi}(e) \\
= & l_{g} \pi_{\psi}\left(\pi_{t}\left(\Phi_{t}(x)\right)\right) \pi_{\psi}(e)=l_{g} \pi_{\psi}\left(\pi_{t}\left(\Phi_{t}(x)\right) e\right) .
\end{aligned}
$$

So if we put $\widetilde{m}_{t}(y):=\widetilde{\pi}_{t}\left(\widetilde{\Phi}_{t}(y)\right)$ we see that

$$
[0, T] \ni t \mapsto \widetilde{m}_{t}\left(l_{g} \pi_{\varphi}(x)\right) \widetilde{e}=l_{g} \pi_{\psi}\left(m_{t}(x) e\right) .
$$

is continuous as $[0, T] \ni t \mapsto m_{t}(x) e$ is continuous. As the span of $l_{g} \pi_{\varphi}(x), x \in B, g \in \mathrm{G}$ is $\sigma$-weakly dense in $\mathcal{O}$ this concludes the second claim.

3.3. Semi-group BMO and interpolation structure. For $x \in \mathcal{M}$ we define the column BMO-semi-norm

$$
\|x\|_{\mathrm{bmo}_{\mathcal{S}}^{c}}=\sup _{t}\left\|\Phi_{t}(x)^{*} \Phi_{t}(x)-\Phi_{t}\left(x^{*} x\right)\right\|^{\frac{1}{2}} .
$$

Then set the row BMO-semi-norm and the BMO-semi-norm by

$$
\|x\|_{\mathrm{bmo}_{\mathcal{S}}^{r}}=\left\|x^{*}\right\|_{\mathrm{bmo}_{\mathcal{S}}^{c}}, \quad\|x\|_{\mathrm{bmo}_{\mathcal{S}}}=\max \left(\|x\|_{\mathrm{bmo}_{\mathcal{S}}^{c}},\|x\|_{\mathrm{bmo}_{\mathcal{S}}^{r}}\right) .
$$

As proved in [JuMe12, Proposition 2.1] these assignments are indeed semi-norms. To proceed further to interpolation we need to treat normed spaces instead and we need to identify BMOspaces as subspaces of $L_{1}(\mathcal{M})$. We can do this using GNS-symmetry and modularity of Markov semi-groups. Note that in [JuMe12] KMS/GNS-symmetry is also part of the standard assumptions on the semi-groups.

Lemma 3.4. We have,

$$
\left\{x \in \mathcal{M} \mid\|x\|_{\text {bmo }_{\mathcal{S}}}=0\right\} \supseteq\left\{x \in \mathcal{M} \mid \forall t \geq 0: \Phi_{t}(x)=x\right\} .
$$

Moreover, if $\mathcal{S}$ is GNS-symmetric then we have equality of these sets. In particular on $\mathcal{M}^{\circ}$ the

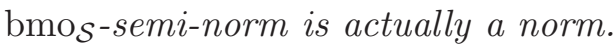

Proof. $\supseteq$. For each $t$ the space of fixed points for $\Phi_{t}$ is a $*$-algebra, see [JuXu07, Remark 7.3]. This shows that if $\Phi_{t}(x)=x$ we also have that

$$
\Phi_{t}\left(x^{*} x\right)-\Phi_{t}(x)^{*} \Phi_{t}(x)=x^{*} x-x^{*} x=0,
$$

and similarly with $x$ replaced by $x^{*}$. That is $\|x\|_{\text {bmo }}=0$.

$\subseteq$. Assume $\mathcal{S}$ is GNS-symmetric. If $\|x\|_{\mathrm{bmo}_{\mathcal{S}}}=0$ (in particular both the row and column BMOsemi-norm is 0) then by [BrOz08, Proposition 1.5.6] we see that $x$ is in the multiplicative domain of $\Phi_{t}$ for every $x \in \mathcal{M}$. We then get for $y \in \mathcal{M}$ that

$$
\varphi(y x)=\varphi\left(\Phi_{t}(y x)\right)=\varphi\left(\Phi_{t}(y) \Phi_{t}(x)\right)=\varphi\left(y \Phi_{2 t}(x)\right),
$$

where the last equality uses $\Phi_{t}$ is GNS-symmetric. This implies that $\Phi_{2 t}(x)=x$ for all $t \geq 0$.

Finally, take $x \in \mathcal{M}^{\circ}$ so $\Phi_{t}(x) \rightarrow 0 \sigma$-weakly. Then, if $\|x\|_{\text {bmos }}=0$ we get by this lemma that for all $t \geq 0$ we have $\Phi_{t}(x)=x$ so that $x=0$.

If $\mathcal{S}$ is $\varphi$-modular GNS-symmetry of $\mathcal{S}$ is equivalent to KMS-symmetry. We prefer to include the KMS-symmetry as part of our statements as all embeddings and interpolation structures are defined with respect to symmetric embeddings. Assume now that $\mathcal{S}$ is $\varphi$-modular and KMSsymmetric. We write bmo $_{\mathcal{S}}^{\circ}$ for the completion of $\mathcal{M}^{\circ}$ equipped with respect to the bmo ${ }_{\mathcal{S}}^{\circ}$-norm. We denote $\operatorname{bmo}_{\mathcal{S}}^{\circ}(\mathcal{M})$ in case we explicitly want to distinguish the von Neumann algebra. 
We now turn bmo $_{\mathcal{S}}^{\circ}$ and into the framework of compatible couples of Banach spaces, see [BeLö76]. Here we really need to restrict ourselves to bmo ${ }_{\mathcal{S}}^{\circ}$ and not just $\mathcal{M}$ with the bmo $\mathcal{S}^{-}$ norm.

Lemma 3.5. Suppose that $A_{2} \geq 0$ is a positive self-adjoint operator on a Hilbert space $H$ so that $\Phi_{t}^{(2)}=\exp \left(-t A_{2}\right)$ is a semi-group of positive contractions on $H$. Suppose that for $\xi \in H$ we have that $\Phi_{t}^{(2)} \xi \rightarrow 0$ weakly as $t \rightarrow \infty$. Then in fact $\Phi_{t}^{(2)} \xi \rightarrow 0$ in the norm of $H$.

Proof. Take a spectral resolution $A_{2}=\int_{0}^{\infty} \lambda d E_{A}(\lambda)$. Let $p_{0}$ be the kernel projection of $A_{2}$ and let $p_{1}=1-p_{0}$. Then $\Phi_{t}^{(2)} \xi \rightarrow 0$ weakly implies that $p_{0} \xi=0$. Now let $p$ be a spectral projection of $A_{2}$ of an interval $\left[\lambda_{0}, \infty\right]$ such that $\|(1-p) \xi\|_{H} \leq \epsilon$. Choose $t_{0} \geq 0$ such that for $t \geq t_{0}$ we have $\left\|\exp \left(-t A_{2}\right) p \xi\right\|_{H} \leq \epsilon$. Then we see $\left\|\exp \left(-t A_{2}\right) \xi\right\|_{H} \leq\left\|\exp \left(-t A_{2}\right) p \xi\right\|_{H}+\| \exp \left(-t A_{2}\right)(1-$ p) $\xi \|_{H} \leq 2 \epsilon$.

Lemma 3.6. Let $\mathcal{S}=\left(\Phi_{t}\right)_{t>0}$ be a $\varphi$-modular, KMS-symmetric, Markov semi-group. Consider BMO-spaces as defined above. We have $\mathrm{bmo}_{\mathcal{S}}^{\circ} \subseteq L_{1}^{\circ}(\mathcal{M})$ through an extension of the embedding $x \mapsto D_{\varphi}^{\frac{1}{2}} x D_{\varphi}^{\frac{1}{2}}$. Moreover, for $x \in \mathcal{M}^{\circ} \subseteq \mathrm{bmo}_{\mathcal{S}}^{\circ}$ we have

$$
\left\|D_{\varphi}^{\frac{1}{2}} x D_{\varphi}^{\frac{1}{2}}\right\|_{1} \leq\|x\|_{\mathrm{bmo}_{\mathcal{S}}^{c}} \quad \text { and } \quad\left\|D_{\varphi}^{\frac{1}{2}} x D_{\varphi}^{\frac{1}{2}}\right\|_{1} \leq\|x\|_{\mathrm{bmo}_{\mathcal{S}}^{r}} .
$$

Proof. $\Phi_{t}^{(2)}$ is a semi-group of positive contractions on $L_{2}(\mathcal{M})$. Further, $\varphi$-modularity of $\mathcal{S}$ implies that $\Phi_{t}^{(2)}\left(x D_{\varphi}^{\frac{1}{2}}\right)=\Phi_{t}(x) D_{\varphi}^{\frac{1}{2}}$ by $(2.2)$. Take $x \in \mathcal{M}^{\circ}$ so that for $y \in \mathcal{M}$

$$
\lim _{t \rightarrow \infty}\left\langle\Phi_{t}(x) D_{\varphi}^{\frac{1}{2}}, y D_{\varphi}^{\frac{1}{2}}\right\rangle=\lim _{t \rightarrow \infty} \varphi\left(y^{*} \Phi_{t}(x)\right) \rightarrow 0 .
$$

This shows that $\Phi_{t}(x) D_{\varphi}^{\frac{1}{2}} \rightarrow 0$ weakly. By Lemma 3.5 then $\left\|\Phi_{t}(x) D_{\varphi}^{\frac{1}{2}}\right\|_{2} \rightarrow 0$. Writing $x=u|x|$ for the polar decomposition we therefore see that

$$
\begin{aligned}
& \left\|D_{\varphi}^{\frac{1}{2}} x D_{\varphi}^{\frac{1}{2}}\right\|_{1}^{2}=\left\|D_{\varphi}^{\frac{1}{2}} u|x| D_{\varphi}^{\frac{1}{2}}\right\|_{1}^{2} \leq\left\|D_{\varphi}^{\frac{1}{2}} u\right\|_{2}^{2}\left\||x| D_{\varphi}^{\frac{1}{2}}\right\|_{2}^{2} \leq\left\||| x \mid D_{\varphi}^{\frac{1}{2}}\right\|_{2}^{2} \\
= & \limsup _{t \rightarrow \infty} \varphi\left(x^{*} x\right)-\left\|\Phi_{t}(x) D_{\varphi}^{\frac{1}{2}}\right\|_{2}^{2}=\limsup _{t \rightarrow \infty} \varphi\left(\Phi_{t}\left(x^{*} x\right)-\Phi_{t}\left(x^{*}\right) \Phi_{t}(x)\right) \\
\leq & \limsup _{t \rightarrow \infty}\left\|\Phi_{t}\left(x^{*} x\right)-\Phi_{t}\left(x^{*}\right) \Phi_{t}(x)\right\|=\sup _{t \geq 0}\left\|\Phi_{t}\left(x^{*} x\right)-\Phi_{t}\left(x^{*}\right) \Phi_{t}(x)\right\|
\end{aligned}
$$

This shows that $\left\|D_{\varphi}^{\frac{1}{2}} x D_{\varphi}^{\frac{1}{2}}\right\|_{1} \leq\|x\|_{\text {bmoc }}$, which yields the claim. As $\left\|D_{\varphi}^{\frac{1}{2}} x D_{\varphi}^{\frac{1}{2}}\right\|_{1}=\left\|D_{\varphi}^{\frac{1}{2}} x^{*} D_{\varphi}^{\frac{1}{2}}\right\|_{1}$

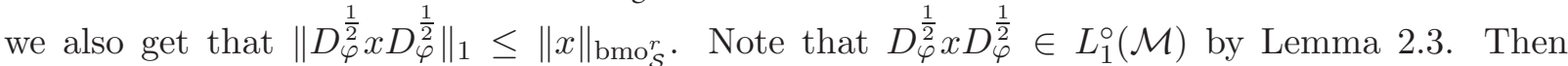
in particular, as by construction $\mathcal{M}^{\circ}$ is dense in $\operatorname{bmo}_{\mathcal{S}}^{\circ}$, we get $\mathrm{bmo}_{\mathcal{S}}^{\circ} \subseteq L_{1}^{\circ}(\mathcal{M})$ through the embedding of the lemma.

We denote the embedding extending $\mathcal{M}^{\circ} \ni x \mapsto D_{\varphi}^{\frac{1}{2}} x D_{\varphi}^{\frac{1}{2}} \in L_{1}^{\circ}(\mathcal{M})$ of Lemma 3.6 by

$$
\kappa_{\mathrm{bmo}}^{\varphi}: \mathrm{bmo}_{\mathcal{S}}^{\circ} \hookrightarrow L_{1}^{\circ}(\mathcal{M}) .
$$

This shows in particular that $\left(\mathrm{bmo}_{\mathcal{S}}^{\circ}, L_{1}^{\circ}(\mathcal{M})\right)$ forms a compatible couple of Banach spaces.

Remark 3.7. We did not consider compatible couples for the case that $\varphi$ is an arbitrary normal, semi-finite, faithful weight; neither this seems obvious. For $L_{p}$-spaces such interpolation structures were explored in [Ter82] and [Izu97].

We recall the following tracial theorem which we will generalize to the non-tracial setting in this paper. 
Theorem 3.8 (Theorem 5.2.(ii) of [JuMe12]). Assume that $\mathcal{M}$ is finite and $\varphi$ is a normal faithful tracial state. Suppose that $\mathcal{S}$ is a KMS-symmetric Markov semi-group. Assume that $\mathcal{S}$ admits a reversed Markov dilation with a.u. continuous path. Then for all $1 \leq p<\infty, 1<q<\infty$ we have

$$
\left[\operatorname{bmo}_{\mathcal{S}}^{\circ}(\mathcal{M}), L_{p}^{\circ}(\mathcal{M})\right]_{\frac{1}{q}}^{\varphi} \approx_{p q} L_{p q}^{\circ}(\mathcal{M}) .
$$

Here $\approx_{p q}$ means complete isomorphism of operator spaces with complete norm of the isomorphism and its inverse bounded by a constant times $p q$.

Remark 3.9. As noted already in [JuMe12, p. 716, after Lemma 4.1], in Theorem 3.8 the condition that $\mathcal{S}$ has a.u. continuous path may be replaced by the weaker condition (see Lemma 2.8 ) that there exists a $\sigma$-weakly dense subset $B \subseteq \mathcal{M}$ such that for every $2 \leq p<\infty$ the martingale $m(x), x \in B$ defined in (2.8) has the property that $\|m(x)\|_{h_{p}^{d}}=0$.

3.4. Interpolation for $\sigma$-finite BMO-spaces. We explicitly record the following lemma here, which is an immediate consequence of complex interpolation, c.f. [BeLö76]. Recall that a subspace $Y$ of a Banach space $X$ is called 1-complemented if there is a norm 1 projection $p: X \rightarrow Y$.

Lemma 3.10. Let $\left(X_{1}, X_{2}\right)$ be a compatible couple of Banach spaces. Let $Y_{i} \subseteq X_{i}$ be 1complemented subspaces. Then $\left(Y_{1}, Y_{2}\right)_{\theta}$ is a 1-complemented subspace of $\left(X_{1}, X_{2}\right)_{\theta}$.

Next we prove that the inclusions of BMO-spaces we need to consider in the proof of Theorem 3.15 are 1-complemented. Both proofs are based on finding Stinespring dilations of the semi-group and the conditional expectation that 'commute' in some sense, c.f. (3.8) and (3.13).

Proposition 3.11. Let $\mathcal{S}$ be a $\varphi$-modular Markov semi-group. We have that $\operatorname{bmo}_{\mathcal{S}}^{\circ}(\mathcal{M})$ is an isometric 1-complemented subspace of $\mathrm{bmo}_{\widetilde{\mathcal{S}}}^{\circ}(\mathcal{R})$.

Proof. As $\widetilde{\mathcal{S}}$ restricts to $\mathcal{S}$ on $\mathcal{M}$ it follows straight from the definition of BMO-spaces that $\operatorname{bmo}_{\mathcal{S}}^{\circ}(\mathcal{M})$ is an isometric subspace of $\operatorname{bmo}_{\widetilde{\mathcal{S}}}^{\circ}(\mathcal{R})$.

We now prove that the conditional expectation $\mathcal{E}_{\mathcal{M}}$ provides a norm 1 projection $\operatorname{bmo}_{\widetilde{\mathcal{S}}}^{\circ}(\mathcal{R}) \rightarrow$ $\operatorname{bmo}_{\mathcal{S}}^{\circ}(\mathcal{M})$. For every $t$ we may take a Stinespring dilation for the ucp map $\Phi_{t}$. That is, there exist a Hilbert space $H$, a contractive map $V_{t}: L_{2}(\mathcal{M}) \rightarrow H$ and a representation $\pi_{t}: \mathcal{M} \rightarrow B(H)$ such that $\Phi_{t}(x)=V_{t}^{*} \pi_{t}(x) V_{t}$. We take amplifications $\widetilde{V}_{t}=1_{\ell_{2}(\mathrm{G})} \otimes V_{t}: \ell_{2}(\mathrm{G}) \otimes L_{2}(\mathcal{M}) \rightarrow \ell_{2}(\mathrm{G}) \otimes H$ and $\widetilde{\pi}_{t}=1_{B\left(\ell_{2}(\mathrm{G})\right)} \otimes \pi_{t}$. Then $\widetilde{\Phi}_{t}(x)=\widetilde{V}_{t}^{*} \widetilde{\pi}_{t}(x) \widetilde{V}_{t}, x \in \mathcal{R}$.

For $\gamma \in \widehat{\mathrm{G}}$ set $W_{\gamma}: \ell_{2}(\mathrm{G}) \rightarrow \ell_{2}(\mathrm{G})$ by $\left(W_{\gamma} \xi\right)(s)=\langle\gamma, s\rangle \xi(s)$. Define a partial isometry

$$
W: \ell_{2}(\mathrm{G}) \rightarrow L_{2}\left(\widehat{\mathrm{G}}, \ell_{2}(\mathrm{G})\right): \xi \mapsto\left(\gamma \mapsto W_{\gamma} \xi\right) .
$$

We may naturally view $W$ as a map $\ell_{2}(\mathrm{G}) \rightarrow \ell_{2}(\mathrm{G}) \otimes L_{2}(\widehat{\mathrm{G}})$. We extend this map to a map $\widetilde{W}: \ell_{2}(\mathrm{G}) \otimes L_{2}(\mathcal{M}) \rightarrow \ell_{2}(\mathrm{G}) \otimes L_{2}(\mathcal{M}) \otimes L_{2}(\widehat{\mathrm{G}})$ as $\widetilde{W}=\Sigma_{23}\left(W \otimes 1_{B\left(L_{2}(\mathcal{M})\right)}\right)$, where $\Sigma_{23}$ flips the second and third tensor coordinate. Then for $x \in \mathcal{R}$ we get that

$$
\widetilde{W}^{*}\left(x \otimes 1_{B\left(L_{2}(\widehat{\mathrm{G}})\right)}\right) \widetilde{W}=\int_{\gamma \in \widehat{\mathrm{G}}} W_{\gamma}^{*} x W_{\gamma} d \gamma=\int_{\gamma \in \widehat{\mathrm{G}}} \theta_{\gamma}(x) d \gamma=\mathcal{E}_{\mathcal{M}}(x) .
$$

That is, $\widetilde{W}$ is a Stinespring dilation for the conditional expectation $\mathcal{E}_{\mathcal{M}}$. We also set $\widetilde{W}^{H}=$ $\Sigma_{23}\left(W \otimes 1_{H}\right)$ as a map $\ell_{2}(\mathrm{G}) \otimes H \rightarrow \ell_{2}(\mathrm{G}) \otimes H \otimes L_{2}(\widehat{\mathrm{G}})$. Note that

$$
\widetilde{W}^{H} \widetilde{V}_{t}=\left(\widetilde{V}_{t} \otimes 1_{L_{2}(\widehat{\mathrm{G}})}\right) \widetilde{W} \text {. }
$$

Further, for $x \in \mathcal{R}$,

$$
\widetilde{\pi}_{t}\left(\widetilde{W}^{*}\left(x \otimes 1_{B\left(L_{2}(\widehat{\mathrm{G}})\right)}\right) \widetilde{W}\right)=\left(\widetilde{W}^{H}\right)^{*}\left(\widetilde{\pi}_{t}(x) \otimes 1_{B\left(L_{2}(\widehat{\mathrm{G}})\right)}\right) \widetilde{W}^{H} .
$$


Now take $x \in \mathcal{R}^{\circ}$. As $\mathcal{E}_{\mathcal{M}}$ is given by (3.2) and $\theta_{\gamma}$ commutes with $\widetilde{\Phi}_{t}$, we get that,

$$
\begin{aligned}
& \Phi_{t}\left(\mathcal{E}_{\mathcal{M}}(x)^{*} \mathcal{E}_{\mathcal{M}}(x)\right)-\Phi_{t}\left(\mathcal{E}_{\mathcal{M}}(x)\right)^{*} \Phi_{t}\left(\mathcal{E}_{\mathcal{M}}(x)\right) \\
= & \Phi_{t}\left(\mathcal{E}_{\mathcal{M}}(x)^{*} \mathcal{E}_{\mathcal{M}}(x)\right)-\mathcal{E}_{\mathcal{M}}\left(\widetilde{\Phi}_{t}(x)^{*}\right) \mathcal{E}_{\mathcal{M}}\left(\widetilde{\Phi}_{t}(x)\right) .
\end{aligned}
$$

Now using the Stinespring dilations and (3.9)

$$
\begin{aligned}
(3.10)= & \widetilde{V}_{t}^{*} \widetilde{\pi}_{t}\left(\widetilde{W}^{*}\left(x^{*} \otimes 1_{B\left(L_{2}(\widehat{\mathrm{G}})\right)}\right) \widetilde{W} \widetilde{W}^{*}\left(x \otimes 1_{B\left(L_{2}(\widehat{\mathrm{G}})\right)}\right) \widetilde{W}\right) \widetilde{V}_{t} \\
& -\widetilde{W}^{*}\left(\widetilde{V}_{t}^{*} \widetilde{\pi}_{t}(x)^{*} \widetilde{V}_{t} \otimes 1_{B\left(L_{2}(\widehat{\mathrm{G}})\right)}\right) \widetilde{W} \widetilde{W}^{*}\left(\widetilde{V}_{t}^{*} \widetilde{\pi}_{t}(x) \widetilde{V}_{t} \otimes 1_{B\left(L_{2}(\widehat{\mathrm{G}})\right)}\right) \widetilde{W} \\
= & \widetilde{V}_{t}^{*}\left(\widetilde{W}^{H}\right)^{*}\left(\widetilde{\pi}_{t}(x)^{*} \otimes 1_{B\left(L_{2}(\widehat{\mathrm{G}})\right)}\right) \widetilde{W}^{H}\left(\widetilde{W}^{H}\right)^{*}\left(\widetilde{\pi}_{t}(x) \otimes 1_{B\left(L_{2}(\widehat{\mathrm{G}})\right)}\right) \widetilde{W}^{H} \widetilde{V}_{t} \\
& -\widetilde{W}^{*}\left(\widetilde{V}_{t}^{*} \widetilde{\pi}_{t}(x)^{*} \widetilde{V}_{t} \otimes 1_{B\left(L_{2}(\widehat{\mathrm{G}})\right)}\right) \widetilde{W} \widetilde{W}^{*}\left(\widetilde{V}_{t}^{*} \widetilde{\pi}_{t}(x) \widetilde{V}_{t} \otimes 1_{B\left(L_{2}(\widehat{\mathrm{G}})\right)}\right) \widetilde{W}
\end{aligned}
$$

Finally we use (3.8) to find,

$$
\begin{aligned}
(3.10)=\widetilde{W}^{*}\left(\widetilde{V}_{t}^{*} \widetilde{\pi}_{t}(x)^{*}\left(\widetilde{V}_{t} \widetilde{V}_{t}^{*}-1\right)^{\frac{1}{2}} \otimes 1_{B\left(L_{2}(\widehat{\mathrm{G}})\right)}\right) \widetilde{W}^{H} & \\
& \times\left(\widetilde{W}^{H}\right)^{*}\left(\left(\widetilde{V_{t}} \widetilde{V}_{t}^{*}-1\right)^{\frac{1}{2}} \widetilde{\pi}_{t}(x) \widetilde{V}_{t} \otimes 1_{B\left(L_{2}(\widehat{\mathrm{G}})\right)}\right) \widetilde{W} .
\end{aligned}
$$

So that

$$
\begin{aligned}
& \left\|\Phi_{t}\left(\mathcal{E}_{\mathcal{M}}(x)^{*} \mathcal{E}_{\mathcal{M}}(x)\right)-\Phi_{t}\left(\mathcal{E}_{\mathcal{M}}(x)\right)^{*} \Phi_{t}\left(\mathcal{E}_{\mathcal{M}}(x)\right)\right\| \\
\leq & \left\|\widetilde{V}_{t}^{*} \widetilde{\pi}_{t}(x)^{*}\left(\widetilde{V}_{t} \widetilde{V}_{t}^{*}-1\right) \widetilde{\pi}_{t}(x) \widetilde{V}_{t} \otimes 1_{B\left(L_{2}(\widehat{\mathrm{G}})\right)}\right\| \\
= & \left\|\widetilde{V}_{t}^{*} \widetilde{\pi}_{t}(x)^{*}\left(\widetilde{V}_{t} \widetilde{V}_{t}^{*}-1\right) \widetilde{\pi}_{t}(x) \widetilde{V}_{t}\right\|=\left\|\widetilde{\Phi}_{t}\left(x^{*} x\right)-\widetilde{\Phi}_{t}(x)^{*} \widetilde{\Phi}_{t}(x)\right\| .
\end{aligned}
$$

Taking the supremum over $t \geq 0$ we get that $\left\|\mathcal{E}_{\mathcal{M}}(x)\right\|_{\mathrm{bmo}_{\mathcal{S}}^{c}} \leq\|x\|_{\mathrm{bmo}}$. By $_{\tilde{\mathcal{S}}}$. Baking adjoints we get the row estimate. By density of $\mathcal{M}^{\circ}$ in $\operatorname{bmo}_{\widetilde{\mathcal{S}}}(\mathcal{R})$ we conclude the proof.

Proposition 3.12. Suppose that $\mathcal{S}$ admits a $\varphi$-modular standard or reversed Markov-dilation. We have that $\mathrm{bmo}_{\widetilde{\mathcal{S}}}^{\circ}\left(\mathcal{R}_{n}\right)$ is an isometric 1-complemented subspace of $\mathrm{bmo}_{\widetilde{\mathcal{S}}}^{\circ}(\mathcal{R})$.

Proof. The conditional expectation of $\mathcal{R}$ onto $\mathcal{R}_{n}$ is given by

$$
\mathcal{F}_{n}(x)=2^{n} \int_{0}^{2^{-n}} \sigma_{s}^{\widetilde{\varphi}_{n}}(x) d s, \quad x \in \mathcal{R},
$$

see [HJX10]. Let $\left(\mathcal{N}_{t}, \pi_{t}, \mathcal{E}_{t}\right)_{t \geq 0}$ be a $\varphi$-modular Markov dilation for $\mathcal{S}$ to a von Neumann algebra $\mathcal{N}$ with normal faithful state $\psi$. By Proposition 3.3 we see that $\widetilde{\mathcal{S}}$ admits a $\widetilde{\varphi}$-modular Markov dilation $\left(\mathcal{O}_{t}, \widetilde{\pi}_{t}, \widetilde{\mathcal{E}}_{t}\right)_{t \geq 0}$. Moreover, the proof shows that we may take $\mathcal{O}_{t}=\mathcal{N}_{t} \rtimes_{\sigma^{\psi}} \mathrm{G}, \widetilde{\mathcal{E}}_{t}=\mathcal{E}_{t} \rtimes_{\sigma^{\psi}} \mathrm{G}$ and $\widetilde{\pi}_{t}=\pi_{t} \rtimes \mathrm{G}$. Let $k_{n}$ be the element in $\mathcal{O}=\cup_{t \geq 0} \mathcal{O}_{t}$ that satisfies $\forall t \geq 0, \widetilde{\pi}_{t}\left(h_{n}\right)=k_{n}$ (formally, it is defined as follows: let $d_{n}=-i \log \left(\lambda_{2^{-n}}\right) \in \mathcal{O}$ (principal branch of the log), then set $e_{n}=2^{n} d_{n}$ and $\left.k_{n}=e^{-d_{n}}\right)$. Set $\widetilde{\psi}_{n}=k_{n} \widetilde{\psi} k_{n}$. The conditional expectation (3.11) may be lifted to the $\mathcal{O}$-level by setting

We get for $x \in \mathcal{R}, t \geq 0$ that

$$
\mathcal{F}_{n}^{\mathcal{O}}(x)=2^{n} \int_{0}^{2^{-n}} \sigma_{s}^{\widetilde{\psi}_{n}}(x) d s, \quad x \in \mathcal{O}
$$

$$
\begin{aligned}
\mathcal{F}_{n}^{\mathcal{O}} \circ \widetilde{\pi}_{t}(x) & =2^{n} \int_{0}^{2^{-n}} \widetilde{\psi}_{s}\left(\widetilde{\pi}_{t}(x)\right) d s=2^{n} \int_{0}^{2^{-n}} k_{n}^{i t} \sigma_{s}^{\widetilde{\psi}}\left(\widetilde{\pi}_{t}(x)\right) k_{n}^{-i t} d s \\
& =2^{n} \int_{0}^{2^{-n}} \widetilde{\pi}_{t}\left(h_{n}^{i t} \sigma_{s}^{\widetilde{\varphi}}(x) h_{n}^{-i t}\right) d s=\widetilde{\pi}_{t} \circ \mathcal{F}_{n}(x) .
\end{aligned}
$$


Recall that $\widetilde{\mathcal{E}}_{t}$ commutes with $\sigma_{s}^{\widetilde{\psi}}$ and has $k_{n}$ in it range so that $\widetilde{\mathcal{E}}_{t}\left(k_{n}^{i s} x k_{n}^{-i s}\right)=k_{n}^{i s} \widetilde{\mathcal{E}}_{t}(x) k_{n}^{-i s}$. Essentially the same computation as before shows that for $x \in \mathcal{O}$ we get that

$$
\begin{aligned}
\mathcal{F}_{n}^{\mathcal{O}} \circ \widetilde{\mathcal{E}}_{t}(x) & \left.=2^{n} \int_{0}^{2^{-n}} \sigma_{s} \widetilde{\psi}_{n}\left(\widetilde{\mathcal{E}}_{t}(x)\right) d s=2^{n} \int_{0}^{2^{-n}} k_{n}^{i s} \sigma_{s}^{\widetilde{\psi}}\left(\widetilde{\mathcal{E}}_{t}(x)\right)\right) k_{n}^{-i s} d s \\
& =2^{n} \int_{0}^{2^{-n}} \widetilde{\mathcal{E}}_{t}\left(k_{n}^{i s} \sigma_{s}^{\widetilde{\psi}}(x) k_{n}^{-i s}\right) d s=\widetilde{\mathcal{E}}_{t} \circ \mathcal{F}_{n}^{\mathcal{O}}(x) .
\end{aligned}
$$

For $x \in \mathcal{R}, t \geq 0$ we get using (2.4) in the second equality and (3.13) in the third,

$$
\begin{aligned}
& \left\|\widetilde{\Phi}_{t}\left(\mathcal{F}_{n}(x)^{*} \mathcal{F}_{n}(x)\right)-\widetilde{\Phi}_{t}\left(\mathcal{F}_{n}(x)\right)^{*} \widetilde{\Phi}_{t}\left(\mathcal{F}_{n}(x)\right)\right\| \\
= & \left\|\widetilde{\pi}_{2 t}\left(\widetilde{\Phi}_{t}\left(\mathcal{F}_{n}(x)^{*} \mathcal{F}_{n}(x)\right)-\widetilde{\Phi}_{t}\left(\mathcal{F}_{n}(x)\right)^{*} \widetilde{\Phi}_{t}\left(\mathcal{F}_{n}(x)\right)\right)\right\| \\
= & \left\|\widetilde{\mathcal{E}}_{2 t}\left(\widetilde{\pi}_{t}\left(\mathcal{F}_{n}(x)^{*} \mathcal{F}_{n}(x)\right)\right)-\widetilde{\mathcal{E}}_{2 t}\left(\widetilde{\pi}_{t}\left(\mathcal{F}_{n}(x)\right)\right)^{*} \widetilde{\mathcal{E}}_{2 t}\left(\widetilde{\pi}_{t}\left(\mathcal{F}_{n}(x)\right)\right)\right\| \\
= & \left\|\widetilde{\mathcal{E}}_{2 t}\left(\mathcal{F}_{n}^{\mathcal{O}}\left(\widetilde{\pi}_{t}(x)^{*}\right) \mathcal{F}_{n}^{\mathcal{O}}\left(\widetilde{\pi}_{t}(x)\right)\right)-\widetilde{\mathcal{E}}_{2 t}\left(\mathcal{F}_{n}^{\mathcal{O}}\left(\widetilde{\pi}_{t}(x)\right)\right)^{*} \widetilde{\mathcal{E}}_{2 t}\left(\mathcal{F}_{n}^{\mathcal{O}}\left(\widetilde{\pi}_{t}(x)\right)\right)\right\| .
\end{aligned}
$$

Next write $P_{2 t}$ and $P_{n}^{\mathcal{O}}$ for the $L_{2}$-implementation of $\widetilde{\mathcal{E}}_{2 t}$ and $\mathcal{F}_{n}^{\mathcal{O}}$ respectively. Then $P_{2 t}$ and $P_{n}^{\mathcal{O}}$ are commuting projections and $\widetilde{\mathcal{E}}_{2 t}(x)=P_{2 t} x P_{2 t}, \mathcal{F}_{n}^{\mathcal{O}}(x)=P_{n}^{\mathcal{O}} x P_{n}^{\mathcal{O}}$. Therefore we may estimate $(3.14)$ as

$$
\begin{aligned}
& \left\|\widetilde{\Phi}_{t}\left(\mathcal{F}_{n}(x)^{*} \mathcal{F}_{n}(x)\right)-\widetilde{\Phi}_{t}\left(\mathcal{F}_{n}(x)\right)^{*} \widetilde{\Phi}_{t}\left(\mathcal{F}_{n}(x)\right)\right\| \\
= & \left\|P_{2 t} P_{n}^{\mathcal{O}} \widetilde{\pi}_{t}(x)^{*} P_{n}^{\mathcal{O}} \widetilde{\pi}_{t}(x) P_{n}^{\mathcal{O}} P_{2 t}-P_{2 t} P_{n}^{\mathcal{O}} \widetilde{\pi}_{t}(x)^{*} P_{n}^{\mathcal{O}} P_{2 t} P_{n}^{\mathcal{O}} \widetilde{\pi}_{t}(x) P_{n}^{\mathcal{O}} P_{2 t}\right\| \\
= & \left\|P_{n}^{\mathcal{O}} P_{2 t} \widetilde{\pi}_{t}(x)^{*}\left(1-P_{2 t}\right) P_{n}^{\mathcal{O}}\left(1-P_{2 t}\right) \widetilde{\pi}_{t}(x) P_{2 t} P_{n}^{\mathcal{O}}\right\| \\
\leq & \left\|P_{2 t} \widetilde{\pi}_{t}(x)^{*}\left(1-P_{2 t}\right) \widetilde{\pi}_{t}(x) P_{2 t}\right\| .
\end{aligned}
$$

By the same computation replacing $\mathcal{F}_{n}(x)$ by just $x$ one gets that

$$
\left\|\widetilde{\Phi}_{t}\left(x^{*} x\right)-\widetilde{\Phi}_{t}(x)^{*} \widetilde{\Phi}_{t}(x)\right\|=\left\|P_{2 t} \widetilde{\pi}_{t}(x)^{*}\left(1-P_{2 t}\right) \widetilde{\pi}_{t}(x) P_{2 t}\right\| .
$$

So that in all we conclude that

$$
\left\|\widetilde{\Phi}_{t}\left(\mathcal{F}_{n}(x)^{*} \mathcal{F}_{n}(x)\right)-\widetilde{\Phi}_{t}\left(\mathcal{F}_{n}(x)\right)^{*} \widetilde{\Phi}_{t}\left(\mathcal{F}_{n}(x)\right)\right\| \leq\left\|\widetilde{\Phi}_{t}\left(x^{*} x\right)-\widetilde{\Phi}_{t}(x)^{*} \widetilde{\Phi}_{t}(x)\right\|
$$

Taking the supremum over all $t \geq 0$ gives $\left\|\mathcal{F}_{n}(x)\right\|_{\mathrm{bmo}_{\tilde{\mathcal{S}}}^{c}\left(\mathcal{R}_{n}\right)} \leq\|x\|_{\mathrm{bmo}_{\tilde{\mathcal{S}}}^{c}(\mathcal{R})}$, which concludes the proof for the column estimate. The row estimate follows by taking adjoints.

Let $\widetilde{\Phi}_{t}^{(p)}$ and $\widetilde{\Phi}_{t}^{(p, n)}$ be the semi-groups acting on $L_{p}(\mathcal{R})$ through interpolation with respect to $\widetilde{\varphi}$ and $\widetilde{\varphi}_{n}$, see $(2.1)$. Note that the definition of the subspace $L_{p}^{\circ}(\mathcal{R})$ of $L_{p}(\mathcal{R})$ depends on the choice of the state. As we are dealing with different states, namely $\widetilde{\varphi}$ and $\widetilde{\varphi}_{n}$ these spaces may in principle be different. We distinghuish this in the notation by writing $L_{p}^{\circ}(\mathcal{R}, \widetilde{\varphi})$ and $L_{p}^{\circ}\left(\mathcal{R}, \widetilde{\varphi}_{n}\right)$. The following proposition shows that the spaces are equal however, so that after it we continue writing $L_{p}^{\circ}(\mathcal{R})$.

Proposition 3.13. Using the notation introduced before Theorem 3.1. Let $1 \leq p<\infty$. We have

$$
D_{\varphi}^{\frac{1}{2 p}}=h_{n}^{-\frac{1}{2 p}} D_{\varphi_{n}}^{\frac{1}{2 p}}=D_{\varphi_{n}}^{\frac{1}{2 p}} h_{n}^{-\frac{1}{2 p}} .
$$

Furthermore, we have for $y \in \mathcal{R}$,

$$
\kappa_{p}^{\widetilde{\varphi}_{n}}(y)=h_{n}^{\frac{1}{2}-\frac{1}{2 p}} \kappa_{p}^{\widetilde{\varphi}}(y) h_{n}^{\frac{1}{2}-\frac{1}{2 p}} .
$$


We have $\widetilde{\Phi}_{t}^{(p, n)}=\widetilde{\Phi}_{t}^{(p)}$ so that in particular $L_{p}^{\circ}(\mathcal{R}, \widetilde{\varphi})=L_{p}^{\circ}\left(\mathcal{R}, \widetilde{\varphi}_{n}\right)$. The same statements hold if $\mathcal{R}$ is replaced by $\mathcal{R}_{n}$.

Proof. (3.15) is an elementary property of spatial derivatives, see [Ter81, Section III]. (3.16) follows as for $y=D_{\widetilde{\varphi}}^{\frac{1}{2 p}} x D_{\widetilde{\varphi}}^{\frac{1}{2 p}}, x \in \mathcal{R}$ we get

$$
\kappa_{p}^{\widetilde{\varphi}}\left(D_{\widetilde{\varphi}}^{\frac{1}{2 p}} x D_{\widetilde{\varphi}}^{\frac{1}{2 p}}\right)=D_{\widetilde{\varphi}}^{\frac{1}{2}} x D_{\widetilde{\varphi}}^{\frac{1}{2}}=h_{n}^{-\frac{1}{2}} D_{\widetilde{\varphi}_{n}}^{\frac{1}{2}} x D_{\widetilde{\varphi}_{n}}^{\frac{1}{2}} h_{n}^{-\frac{1}{2}}=h_{n}^{-\frac{1}{2}+\frac{1}{2 p}} \kappa_{p}^{\widetilde{\varphi}_{n}}(y) h_{n}^{-\frac{1}{2}+\frac{1}{2 p}} .
$$

By using the definitions and Lemma 3.2 we see that for $x \in \mathcal{R}$ we get

$$
\begin{aligned}
& \widetilde{\Phi}_{t}^{(p, n)}\left(D_{\widetilde{\varphi}}^{\frac{1}{2 p}} x D_{\widetilde{\varphi}}^{\frac{1}{2 p}}\right)=\widetilde{\Phi}_{t}^{(p, n)}\left(D_{\widetilde{\varphi}_{n}}^{\frac{1}{2 p}} h_{n}^{-\frac{1}{2 p}} x h_{n}^{-\frac{1}{2 p}} D_{\widetilde{\varphi}_{n}}^{\frac{1}{2 p}}\right)=D_{\widetilde{\varphi}_{n}}^{\frac{1}{2 p}} \widetilde{\Phi}_{t}^{(p)}\left(h_{n}^{-\frac{1}{2 p}} x h_{n}^{-\frac{1}{2 p}}\right) D_{\widetilde{\varphi}_{n}}^{\frac{1}{2 p}} \\
= & D_{\widetilde{\varphi}_{n}}^{\frac{1}{2 p}} h_{n}^{-\frac{1}{2 p}} \widetilde{\Phi}_{t}^{(p)}(x) h_{n}^{-\frac{1}{2 p}} D_{\widetilde{\varphi}_{n}}^{\frac{1}{2 p}}=D_{\widetilde{\varphi}}^{\frac{1}{2 p}} \widetilde{\Phi}_{t}^{(p)}(x) D_{\widetilde{\varphi}}^{\frac{1}{2 p}}=\widetilde{\Phi}_{t}^{(p)}\left(D_{\widetilde{\varphi}}^{\frac{1}{2 p}} x D_{\widetilde{\varphi}}^{\frac{1}{2 p}}\right) .
\end{aligned}
$$

This shows by density that $\widetilde{\Phi}_{t}^{(p, n)}=\widetilde{\Phi}_{t}^{(p)}$.

Now consider compatible couples $\left[\mathrm{bmo}_{\widetilde{\mathcal{S}}}^{\circ}(\mathcal{R}), L_{p}^{\circ}(\mathcal{R})\right]^{\widetilde{\varphi}}$ and $\left[\mathrm{bmo}_{\widetilde{\mathcal{S}}}^{\circ}(\mathcal{R}), L_{p}^{\circ}(\mathcal{R})\right]^{\widetilde{\varphi}_{n}}$ with respect to respective states $\widetilde{\varphi}$ and $\widetilde{\varphi}_{n}$. Note that $\mathcal{R}^{\circ}$ is by definition contained in $\operatorname{bmo}_{\widetilde{\mathcal{S}}}^{\circ}(\mathcal{R})$. Let $\kappa_{[\mathrm{bmo}, p ; q]}^{\widetilde{\varphi}}$ and $\kappa_{[\mathrm{bmo}, p ; q]}^{\widetilde{\varphi}_{n}}$ be the respective natural identifications of $\left[\mathrm{bmo}_{\widetilde{\mathcal{S}}}^{\circ}(\mathcal{R}), L_{p}^{\circ}(\mathcal{R})\right]_{1 / q}^{\widetilde{\varphi}}$ and $\left[\mathrm{bmo}_{\widetilde{\mathcal{S}}}^{\circ}(\mathcal{R}), L_{p}^{\circ}(\mathcal{R})\right]_{1 / q}^{\widetilde{\varphi}_{n}}$ as subspaces of $L_{1}(\mathcal{R})$.

Proposition 3.14. Let $\mathcal{S}$ be a $\varphi$-modular KMS-symmetric semi-group. We have a complete isometry

$$
\sigma_{p, q, n}:\left[\mathrm{bmo}_{\widetilde{\mathcal{S}}}^{\circ}(\mathcal{R}), L_{p}^{\circ}(\mathcal{R})\right]_{1 / q}^{\widetilde{\varphi}} \rightarrow\left[\mathrm{bmo}_{\widetilde{\mathcal{S}}}^{\circ}(\mathcal{R}), L_{p}^{\circ}(\mathcal{R})\right]_{1 / q}^{\widetilde{\varphi}_{n}}
$$

Moreover, the isometry is explicitly given by

$$
\kappa_{[\mathrm{bmo}, p ; q]}^{\widetilde{\varphi}_{n}} \circ \sigma_{p, q, n}(y)=h_{n}^{\frac{1}{2}-\frac{1}{2 p q}} \kappa_{[\mathrm{bmo}, p ; q]}^{\widetilde{\varphi}}(y) h_{n}^{\frac{1}{2}-\frac{1}{2 p q}} .
$$

Proof. We use short hand notation $X=\kappa_{\text {bmo }}^{\widetilde{\varphi}}\left(\operatorname{bmo}_{\widetilde{\mathcal{S}}}^{\circ}(\mathcal{R})\right), X_{n}=\kappa_{\text {bmo }}^{\widetilde{\varphi}_{n}}\left(\mathrm{bmo}_{\widetilde{\mathcal{S}}}^{\circ}(\mathcal{R})\right), Y=\kappa_{p}^{\widetilde{\varphi}}\left(L_{p}^{\circ}(\mathcal{R})\right)$ and $Y_{n}=\kappa_{p}^{\widetilde{\varphi}_{n}}\left(L_{p}^{\circ}(\mathcal{R})\right)$. The norm on $X$ and $X_{n}$ is just the norm of bmo ${ }_{\widetilde{\mathcal{S}}}^{\circ}(\mathcal{R})$ through the respective embeddings $\kappa_{\text {bmo }}^{\widetilde{\varphi}}$ and $\kappa_{\text {bmo }}^{\widetilde{\varphi}_{n}}$. Similarly the norms on $Y$ and $Y_{n}$ is just the norm of $L_{p}(\mathcal{R})$. Let $\sigma_{n}$ be the map $\left[\operatorname{bmo}_{\widetilde{\mathcal{S}}}^{\circ}(\mathcal{R}), L_{p}^{\circ}(\mathcal{R})\right]_{\frac{1}{q}}^{\widetilde{\varphi}} \rightarrow L_{1}^{\circ}(\mathcal{R})$ defined by

$$
\sigma_{n}\left(\kappa_{[\mathrm{bmo}, p ; q]}^{\widetilde{\varphi}}(y)\right)=h_{n}^{\frac{1}{2}-\frac{1}{2 p q}} \kappa_{[\mathrm{bmo}, p ; q]}^{\widetilde{\varphi}}(y) h_{n}^{\frac{1}{2}-\frac{1}{2 p q}},
$$

i.e. the mapping (3.17) on the $L_{1}$-level.

Take $f \in \mathcal{F}(X, Y)$ which we view as a function on the strip $S \rightarrow X+Y$, where $X+Y$ is a (non-isometric) subspace of $L_{1}(\mathcal{R})$, see Section 2.2. Define

$$
\left(U_{n} f\right)(z)=h_{n}^{\frac{i z}{2 p}+\frac{1}{2}} f(z) h_{n}^{\frac{i z}{2 p}+\frac{1}{2}} \in L_{1}^{\circ}(\mathcal{R}), \quad z \in S .
$$

We claim that $U_{n} f \in \mathcal{F}\left(X_{n}, Y_{n}\right)$. Take $s \in \mathbb{R}$ so that by definition of $\mathcal{F}(X, Y), f(s)=\kappa_{\text {bmo }}^{\widetilde{\varphi}}(x)$ for some $x \in \operatorname{bmo}_{\widetilde{S}}^{\circ}(\mathcal{R})$. Then by (3.7)

$$
\left(U_{n} f\right)(s)=h_{n}^{\frac{i s}{2 p}+\frac{1}{2}} f(s) h_{n}^{\frac{i s}{2 p}+\frac{1}{2}}=\kappa_{\mathrm{bmo}}^{\widetilde{\varphi}_{n}}\left(h_{n}^{\frac{i s}{2 p}} x h_{n}^{\frac{i s}{2 p}}\right) .
$$


Further, for $y \in \mathcal{R}^{\circ}$ it follows from the definition of the BMO-norm and Lemma 3.2 that

$$
\begin{aligned}
& \left.\left\|h_{n}^{\frac{i s}{2 p}} y h_{n}^{\frac{i s}{2 p}}\right\|_{\mathrm{bmo}}^{2}=\sup _{\tilde{\mathcal{S}}} \| \widetilde{\Phi}_{t}\left(\left(h_{n}^{\frac{i s}{2 p}} y h_{n}^{\frac{i s}{2 p}}\right)^{*}\left(h_{n}^{\frac{i s}{2 p}} y h_{n}^{\frac{i s}{2 p}}\right)\right)-\widetilde{\Phi}_{t}\left(h_{n}^{\frac{i s}{2 p}} y h_{n}^{\frac{i s}{2 p}}\right)^{*} \widetilde{\Phi}_{t}\left(h_{n}^{\frac{i s}{2 p}} y h_{n}^{\frac{i s}{2 p}}\right)\right) \| \\
& =\sup _{t \geq 0}\left\|h_{n}^{-\frac{i s}{2 p}} \widetilde{\Phi}_{t}\left(y^{*} y\right) h_{n}^{\frac{i s}{2 p}}-h_{n}^{-\frac{i s}{2 p}} \widetilde{\Phi}_{t}(y)^{*} \widetilde{\Phi}_{t}(y) h_{n}^{\frac{i s}{2 p}}\right\|=\|y\|_{\mathrm{bmo}_{\tilde{\mathcal{S}}}^{c}}^{2} .
\end{aligned}
$$

The same holds for the row BMO-space so that $\left\|h_{n}^{\frac{i s}{2 p}} y h_{n}^{\frac{i s}{2 p}}\right\|_{\mathrm{bmo}_{\tilde{S}}}^{2}=\|y\|_{\mathrm{bmo} \tilde{s}}^{2}$. And by density this in fact holds for all $y \in \operatorname{bmo}_{\widetilde{\mathcal{S}}}^{\circ}(\mathcal{R})$. This shows that for $s \in \mathbb{R}$ we get $\left(U_{n} f\right)(s) \in X_{n}$ and

$$
\left\|\left(U_{n} f\right)(s)\right\|_{\mathrm{bmo}_{\tilde{\mathcal{S}}}}=\|f(s)\|_{\mathrm{bmo}_{\tilde{\mathcal{S}}}} .
$$

Next consider $i+s \in i+\mathbb{R}$. By definition of $\mathcal{F}(X, Y)$ we have $f(i+s) \in Y$, so write $f(i+s)=$ $\kappa_{p}^{\widetilde{\varphi}}(x)$ for $x \in L_{p}^{\circ}(\mathcal{R})$. Then from (3.3) and (3.16)

$$
\left(U_{n} f\right)(i+s)=h_{n}^{\frac{i s}{2 p}-\frac{1}{2 p}+\frac{1}{2}} f(s) h_{n}^{\frac{i s}{2 p}-\frac{1}{2 p}+\frac{1}{2}}=h_{n}^{\frac{i s}{2 p}} \kappa_{p}^{\widetilde{\varphi}_{n}}(x) h_{n}^{\frac{i s}{2 p}}=\kappa_{p}^{\widetilde{\varphi}_{n}}\left(h_{n}^{\frac{i s}{2 p}} x h_{n}^{\frac{i s}{2 p}}\right) .
$$

Proposition 3.13 shows then that $\left(U_{n} f\right)(i+s) \in Y_{n}$ and

$$
\left\|\left(U_{n} f\right)(i+s)\right\|_{p}=\|f(i+s)\|_{p} .
$$

We get from the equations (3.18) and (3.20) that $U_{n} f \in \mathcal{F}\left(X_{n}, Y_{n}\right)$ as the fact that $h_{n}$ is boundedly invertible implies that $U_{n} f$ is continuous on the strip $S$ and analytic on its interior. Moreover, $\left\|U_{n} f\right\|_{\mathcal{F}\left(X_{n}, Y_{n}\right)} \leq\|f\|_{\mathcal{F}(X, Y)}$. So the assignment $f \mapsto U_{n} f$ is a contraction. Consider for $f \in \mathcal{F}\left(X_{n}, Y_{n}\right)$ the function

$$
\left(V_{n} f\right)(z)=h_{n}^{-\frac{i z}{2 p}-\frac{1}{2}} f(z) h_{n}^{-\frac{i z}{2 p}-\frac{1}{2}}, \quad z \in S
$$

Then exactly as in the previous paragraph one proves that $V_{n} f \in \mathcal{F}(X, Y)$ and $\left\|V_{n} f\right\|_{\mathcal{F}(X, Y)} \leq$ $\|f\|_{\mathcal{F}\left(X_{n}, Y_{n}\right)}$. Moreover $V_{n}=U_{n}^{-1}$ and hence $\mathcal{F}(X, Y)$ and $\mathcal{F}\left(X_{n}, Y_{n}\right)$ are isometrically isomorphic.

Now take $x \in[X, Y]_{1 / q}$. Let $\epsilon>0$. Take $f \in \mathcal{F}(X, Y)$ such that $f\left(\frac{i}{q}\right)=x$ and $\|x\|_{[X, Y]_{1 / q}} \leq$ $\|f\|_{\mathcal{F}(X, Y)}+\epsilon$. Then $\sigma_{n}(x)=\left(U_{n} f\right)\left(\frac{i}{q}\right)$ so that $\left\|\sigma_{n}(x)\right\|_{\left[X_{n}, Y_{n}\right]_{1 / q}} \leq\left\|U_{n} f\right\|_{\mathcal{F}\left(X_{n}, Y_{n}\right)}=\|f\|_{\mathcal{F}(X, Y)} \leq$ $\|x\|_{[X, Y]_{1 / q}}+\epsilon$. This shows that the map (3.17) is well-defined and contractive. Repeating this argument for $V_{n}$ instead of $U_{n}$ shows that in fact (3.17) is an isometric isomorphism. That the map is completely isometric follows by repeating the argument on matrix levels.

Theorem 3.15. Let $(\mathcal{M}, \varphi)$ be a von Neumann algebra with normal faithful state. Let $\mathcal{S}=\left(\Phi_{t}\right)_{t \geq 0}$ be a $\varphi$-modular KMS-symmetric Markov semi-group. Assume that $\mathcal{S}$ admits a reversed Markov dilation with a.u. continuous path. Then we have, for all $1 \leq p<\infty, 1<q<\infty$,

$$
\left[\operatorname{bmo}_{\mathcal{S}}^{\circ}(\mathcal{M}), L_{p}^{\circ}(\mathcal{M})\right]_{1 / q} \approx_{p q} L_{p q}^{\circ}(\mathcal{M}) .
$$

Proof. Because $\mathcal{S}=\left(\Phi_{t}\right)_{t \geq 0}$ is $\varphi$-modular it may be extended to Markov semi-group $\widetilde{\mathcal{S}}=\left(\widetilde{\Phi}_{t}\right)_{t \geq 0}$ on $\mathcal{R}$, see Lemma 3.2. By Proposition $3.3 \widetilde{\mathcal{S}}$ also has a reversed Markov dilation with a.u. continuous path. We claim that this map preserves $\mathcal{R}_{n}:=\mathcal{R}_{\widetilde{\varphi}_{n}}$, which was defined as the centralizer of $\widetilde{\varphi}_{n}$. Let $x \in \mathcal{R}_{n}$. Then, by applying [Tak03, Theorem VIII.3.3] twice and Lemma 3.2 we get,

$$
\sigma_{s}^{\widetilde{\varphi}_{n}}\left(\widetilde{\Phi}_{t}(x)\right)=h_{n}^{i s} \sigma_{s}^{\widetilde{\varphi}}\left(\widetilde{\Phi}_{t}(x)\right) h_{n}^{-i s}=h_{n}^{i s} \widetilde{\Phi}_{t}\left(\sigma_{s}^{\widetilde{\varphi}}(x)\right) h_{n}^{-i s}=\widetilde{\Phi}_{t}\left(h_{n}^{i s} \sigma_{s}^{\widetilde{\varphi}}(x) h_{n}^{-i s}\right)=\widetilde{\Phi}_{t}(x) .
$$

So that $x \in \mathcal{R}_{n}$. Denote the restriction of $\widetilde{\Phi}_{t}$ to $\mathcal{R}_{n}$ by $\widetilde{\Phi}_{n, t}$. In all, we obtained Markov semigroups $\widetilde{\mathcal{S}}=\left(\widetilde{\Phi}_{t}\right)_{t \geq 0}$ and $\widetilde{\mathcal{S}}_{n}=\left(\widetilde{\Phi}_{n, t}\right)_{t \geq 0}$ with respect to the respective states $\widetilde{\varphi}$ and $\left.\widetilde{\varphi}\right|_{\mathcal{R}_{n}}$. Note 
that by Lemma 3.2

$$
\widetilde{\varphi}_{n} \circ \widetilde{\Phi}_{t}(x)=\widetilde{\varphi}\left(h_{n}^{\frac{1}{2}} \widetilde{\Phi}_{t}(x) h_{n}^{\frac{1}{2}}\right)=\widetilde{\varphi}\left(\widetilde{\Phi}_{t}\left(h_{n}^{\frac{1}{2}} x h_{n}^{\frac{1}{2}}\right)=\widetilde{\varphi}\left(h_{n}^{\frac{1}{2}} x h_{n}^{\frac{1}{2}}\right)=\widetilde{\varphi}_{n}(x) .\right.
$$

This shows that $\widetilde{\Phi}_{t}: \mathcal{R}_{n} \rightarrow \mathcal{R}_{n}$ is also Markov with respect to $\widetilde{\varphi}_{n}$, which is tracial on $\mathcal{R}_{n}$.

As the semi-groups $\mathcal{S}$ and $\widetilde{\mathcal{S}}_{n}$ are restrictions of $\widetilde{\mathcal{S}}$ we have isometric inclusions of the corresponding BMO-spaces

$$
\operatorname{bmo}_{\mathcal{S}}^{\circ}(\mathcal{M}) \subseteq \operatorname{bmo}_{\widetilde{\mathcal{S}}}^{\circ}(\mathcal{R}), \quad \operatorname{bmo}_{\widetilde{\mathcal{S}}_{n}}^{\circ}\left(\mathcal{R}_{n}\right) \subseteq \operatorname{bmo}_{\widetilde{\mathcal{S}}}^{\circ}(\mathcal{R}), \quad n \in \mathbb{N} .
$$

Moreover, these inclusions are 1-complemented by Lemmas 3.11 and 3.12. Lemma 3.2 also shows that $\widetilde{\mathcal{S}}$ admits a reversed Markov dilation with a.u. continuous path. Moreover, this dilation may be chosen to be a dilation with respect to $\widetilde{\varphi}_{n}$. Let $m(x)=\left(m_{t}(x)\right)_{t>0}$ be the martingale with $x$ in the set $B$ described in Definition 2.9 for this Markov dilation. By Lemma 2.8 we see that for every $2 \leq p<\infty$ we have $\|m(x)\|_{h_{p}^{d}}=0$ and then by $(2.7)$ we see that $\left\|m\left(\mathcal{F}_{n}(x)\right)\right\|_{h_{p}^{d}}=0$. This shows that $\mathcal{F}_{n}(B)$ is a $\sigma$-weakly dense subset of $\mathcal{R}_{n}$ such that the martingale $m(x), x \in \mathcal{F}_{n}(B)$ has vanishing $h_{p}^{d}$-norm. Therefore, by Remark 3.9 the Theorem 3.8 applies to the von Neumann algebra $\mathcal{R}_{n}$ with normal tracial state $\widetilde{\varphi}_{n}$ with Markov semi-group $\widetilde{\mathcal{S}}_{n}$.

So Theorem 3.8 yields

$$
\left[\operatorname{bmo}_{\mathcal{S}_{n}}^{\circ}\left(\mathcal{R}_{n}\right), L_{q}^{\circ}\left(\mathcal{R}_{n}\right)\right]_{1 / p}^{\widetilde{\varphi}_{n}} \approx_{p q} L_{p q}\left(\mathcal{R}_{n}\right)^{\circ}
$$

Now we have isometries

$$
\begin{aligned}
& {\left[\mathrm{bmo}_{\widetilde{\mathcal{S}}_{n}}^{\circ}\left(\mathcal{R}_{n}\right), L_{p}^{\circ}\left(\mathcal{R}_{n}\right)\right]_{1 / q}^{\widetilde{\varphi}_{n}} \stackrel{\text { Lemma } 3.10}{\longrightarrow}\left[\mathrm{bmo}_{\widetilde{\mathcal{S}}}^{\circ}(\mathcal{R}), L_{p}^{\circ}(\mathcal{R})\right]_{1 / q}^{\widetilde{\varphi}_{n}}} \\
& \approx_{p q} \uparrow \quad \mid \sigma_{p, q, n}^{-1} \quad \text { Prop. } 4.4 \\
& L_{p q}^{\circ}\left(\mathcal{R}_{n}\right) \quad\left[\mathrm{bmo}_{\mathcal{S}}^{\circ}(\mathcal{R}), L_{p}^{\circ}(\mathcal{R})\right]_{1 / q}^{\widetilde{\varphi}} .
\end{aligned}
$$

Furthermore, for $x \in \mathcal{R}_{n}$,

$$
\begin{aligned}
& \kappa_{[\mathrm{bmo}, p ; q]}^{\widetilde{\varphi}} \circ \sigma_{p, q, n}^{-1}\left(D_{\widetilde{\varphi}}^{\frac{1}{2 p q}} x D_{\widetilde{\varphi}}^{\frac{1}{2 p q}}\right)=h_{n}^{-\frac{1}{2}+\frac{1}{2 p q}} \kappa_{[\mathrm{bmo}, p ; q]}^{\widetilde{\varphi}_{n}}\left(D_{\widetilde{\varphi}}^{\frac{1}{2 p q}} x D_{\widetilde{\varphi}}^{\frac{1}{2 p q}}\right) h_{n}^{-\frac{1}{2}+\frac{1}{2 p q}} \\
= & h_{n}^{-\frac{1}{2}+\frac{1}{2 p q}} \kappa_{[\mathrm{bmo}, p ; q]}^{\widetilde{\varphi}_{n}}\left(D_{\widetilde{\varphi_{n}}}^{\frac{1}{2 p q}} h_{n}^{-\frac{1}{2 p q}} x h_{n}^{-\frac{1}{2 p q}} D_{\widetilde{\varphi}_{n}}^{\frac{1}{2 p q}}\right) h_{n}^{-\frac{1}{2}+\frac{1}{2 p q}} \\
= & \left.h_{n}^{-\frac{1}{2}+\frac{1}{2 p q}} D_{\widetilde{\varphi_{n}}}^{\frac{1}{2}} h_{n}^{-\frac{1}{2 p q}} x h_{n}^{-\frac{1}{2 p q}} D_{\widetilde{\varphi_{n}}}^{\frac{1}{2}}\right) h_{n}^{-\frac{1}{2}+\frac{1}{2 p q}} \\
= & D_{\widetilde{\varphi}}^{\frac{1}{2}} x D_{\widetilde{\varphi}}^{\frac{1}{2}} .
\end{aligned}
$$

It follows that for each $n \in \mathbb{N}$ we have an isometric embedding,

$$
j_{n}: L_{p q}^{\circ}\left(\mathcal{R}_{n}\right) \rightarrow\left[\operatorname{bmo}_{\widetilde{S}}^{\circ}(\mathcal{R}), L_{p}^{\circ}(\mathcal{R})\right]_{1 / q}^{\widetilde{\varphi}},
$$

and these embeddings are compatible with the inclusions $L_{p q}^{\circ}\left(\mathcal{R}_{n}\right) \subseteq L_{p q}^{\circ}\left(\mathcal{R}_{n+1}\right)$ with respect to $\widetilde{\varphi}$. This shows that $\cup_{n \in \mathbb{N}} L_{p q}^{\circ}\left(\mathcal{R}_{n}, \widetilde{\varphi}\right)$ can isometrically be identified with a subspace of $\left[\operatorname{bmo}_{\widetilde{S}}(\mathcal{R}), L_{p}^{\circ}(\mathcal{R})\right]_{1 / q}^{\widetilde{\varphi}}$. As $\cup_{n \in \mathbb{N}} L_{p q}^{\circ}\left(\mathcal{R}_{n}\right)$ is dense in $L_{p q}^{\circ}(\mathcal{R})$, c.f. [Gol84, Theorem 8], we see that $L_{p q}^{\circ}(\mathcal{R})$ is isometrically contained in the space $\left[\mathrm{bmo}_{\widetilde{\mathcal{S}}}(\mathcal{R}), L_{p}^{\circ}(\mathcal{R})\right]_{1 / q}^{\widetilde{\varphi}}$. By $\left[\right.$ BeLö76, Theorem 4.2.2.(a)] we have that $\mathcal{R}^{\circ}$ is dense in $\left[\mathrm{bmo}_{\widetilde{\mathcal{S}}}^{\circ}(\mathcal{R}), L_{q}^{\circ}(\mathcal{R})\right]_{1 / p}^{\widetilde{\varphi}}$. Further as $\mathcal{R}^{\circ}$ is also contained in $L_{p q}^{\circ}(\mathcal{R})$ we must have an isomorphism

$$
\left[\mathrm{bmo}_{\mathcal{S}_{n}}^{\circ}(\mathcal{R}), L_{q}^{\circ}(\mathcal{R})\right]_{1 / p} \approx_{p q} L_{p q}^{\circ}(\mathcal{R})
$$


Now again by Lemma 3.10 we see that the space $\left[\operatorname{bmo}_{\mathcal{S}}^{\circ}(\mathcal{M}), L_{p}^{\circ}(\mathcal{M})\right]_{1 / q}^{\varphi}$ is a 1-complemented subspace of the left hand side of (3.21) and hence of $L_{p q}^{\circ}(\mathcal{R})$. Further by [BeLö76, Theorem 4.2.2.(a)] the space $\left[\mathrm{bmo}_{\mathcal{S}_{n}}^{\circ}(\mathcal{M}), L_{p}^{\circ}(\mathcal{M})\right]_{1 / q}^{\varphi}$ contains $\mathcal{M}^{\circ}$ densely. Since in turn $\mathcal{M}^{\circ}$ is dense in $L_{p q}^{\circ}(\mathcal{M})$ which is included in $L_{p q}^{\circ}(\mathcal{R})$ isometrically, we conclude that $\left[\operatorname{bmo}_{\mathcal{S}_{n}}^{\circ}(\mathcal{M}), L_{q}(\mathcal{M})^{\circ}\right]_{1 / p}^{\varphi} \approx_{p q}$ $L_{p q}^{\circ}(\mathcal{M})$. Isomorphisms holds for complete bounds by considering matrix levels.

\section{Other BMO-Spaces associated With Markov Semi-Groups}

As in the rest of this paper let $\mathcal{M}$ be von Neumann algebra with faithful normal state $\varphi$. Let $\mathcal{S}=\left(\Phi_{t}\right)_{t \geq 0}$ be a Markov semi-group on $\mathcal{M}$. Define a semi-norm (see [JuMe12, Proposition 2.1]),

$$
\|x\|_{\mathrm{BMO}_{\mathcal{S}}^{c}}=\sup _{t \geq 0}\left\|\Phi_{t}\left(\left|x-\Phi_{t}(x)\right|^{2}\right)\right\|^{\frac{1}{2}}
$$

and then set

$$
\|x\|_{\mathrm{BMO}_{\mathcal{S}}^{r}}=\left\|x^{*}\right\|_{\mathrm{BMO}_{\mathcal{S}}^{c}}, \quad\|x\|_{\mathrm{BMO}_{\mathcal{S}}}=\max \left(\|x\|_{\mathrm{BMO}_{\mathcal{S}}^{c}},\|x\|_{\mathrm{BMO}_{\mathcal{S}}^{r}}\right) .
$$

Lemma 4.1. For $x \in \mathcal{M}$ we have $\|x\|_{\mathrm{BMO}_{\mathcal{S}}}=0$ if and only if for all $t \geq 0, \Phi_{t}(x)=x$.

Proof. The if part is obvious. Conversly, if $\|x\|_{\mathrm{BMO}_{\mathcal{S}}}=0$ then for all $t \geq 0$ we have $\| \Phi_{t}(\mid x-$ $\left.\left.\Phi_{t}(x)\right|^{2}\right) \|=0$ and so $0=\varphi\left(\Phi_{t}\left(\left|x-\Phi_{t}(x)\right|^{2}\right)\right)=\varphi\left(\left|x-\Phi_{t}(x)\right|^{2}\right)$. As $\varphi$ is faithful $x=\Phi_{t}(x)$.

We see that on $\mathcal{M}^{\circ}$ the BMO-semi-norm is actually a norm and its completion will be denoted by $\mathrm{BMO}_{\mathcal{S}}^{\circ}$ or $\mathrm{BMO}_{\mathcal{S}}^{\circ}(\mathcal{M})$. Note that we do not need to assume KMS-symmetry here.

Furthermore, let $A_{2}$ be the closed densely defined operator such that $\exp \left(-t A_{2}\right)=\Phi_{t}^{(2)}, t \geq 0$, see Section 2.3. The Poisson semi-group $\mathcal{P}=\left(\Psi_{t}\right)_{t \geq 0}$ is defined as the unique Markov semi-group such that $\Psi_{t}^{(2)}=\exp \left(-t A_{2}^{\frac{1}{2}}\right), t \geq 0$ (see [Sau99]). Therefore we obtain BMO-spaces

$$
\mathrm{bmo}_{\mathcal{P}}^{\circ}=\operatorname{bmo}_{\mathcal{P}}^{\circ}(\mathcal{M}), \quad \mathrm{BMO}_{\mathcal{P}}^{\circ}=\operatorname{BMO}_{\mathcal{P}}^{\circ}(\mathcal{M}),
$$

together with their obvious row and column counterparts. Then [JuMe12, Theorem 5.2] proves the following tracial interpolation result.

Theorem 4.2. Let $\mathcal{M}$ be a von Neumann algebra with faithful normal tracial state $\varphi$. Let $\mathcal{S}=\left(\Phi_{t}\right)_{t \geq 0}$ be a KMS-symmetric Markov semi-group for $(\mathcal{M}, \varphi)$. Assume that $\mathcal{S}$ admits a standard Markov dilation. Then,

$$
\left[X, L_{p}^{\circ}(\mathcal{M})\right]_{1 / q} \approx_{p q} L_{p q}^{\circ}(\mathcal{M})
$$

where $X$ is any of the spaces $\mathrm{BMO}_{\mathcal{S}}^{\circ}(\mathcal{M}), \operatorname{bmo}_{\mathcal{P}}^{\circ}(\mathcal{M})$ or $\mathrm{BMO}_{\mathcal{P}}^{\circ}(\mathcal{M})$.

We may generalize this to the non-tracial setting in the following way. The proof follows closely the lines of Theorem 3.15. We give the main differences. Firstly, we have that $\mathrm{BMO}_{\mathcal{S}}^{\circ}(\mathcal{M})$ embeds contractively into $L_{1}(\mathcal{M})$ as for $x \in \mathcal{M}^{\circ}$ with polar decomposition $x=u|x|$ we get that

$$
\begin{aligned}
\left\|D_{\varphi}^{\frac{1}{2}} x D_{\varphi}^{\frac{1}{2}}\right\|_{1}^{2} & =\left\|D_{\varphi}^{\frac{1}{2}} u|x| D_{\varphi}^{\frac{1}{2}}\right\|_{1}^{2} \leq\left\|D_{\varphi}^{\frac{1}{2}} u\right\|_{2}^{2}\left\||x| D_{\varphi}^{\frac{1}{2}}\right\|_{2}^{2} \leq\left\|D_{\varphi}^{\frac{1}{2}} x^{*} x D_{\varphi}^{\frac{1}{2}}\right\|_{1}=\varphi\left(x^{*} x\right) \\
& =\lim _{t \rightarrow \infty} \varphi\left(x^{*} x+\Phi_{t}(x)^{*} \Phi_{t}(x)-\Phi_{t}(x)^{*} x-x^{*} \Phi_{t}(x)\right) \\
& =\lim _{t \rightarrow \infty} \varphi\left(\Phi_{t}\left(x^{*} x+\Phi_{t}(x)^{*} \Phi_{t}(x)-\Phi_{t}(x)^{*} x-x^{*} \Phi_{t}(x)\right)\right) \\
& \leq \limsup _{t \rightarrow \infty} \varphi\left(\Phi_{t}\left(x^{*} x+\Phi_{t}(x)^{*} \Phi_{t}(x)-\Phi_{t}(x)^{*} x-x^{*} \Phi_{t}(x)\right)\right) \leq\|x\|_{\mathrm{BMO}_{\mathcal{S}}^{\circ}} .
\end{aligned}
$$

A similar argument holds for the row estimate, which yields a version of Lemma 3.6 for $\mathrm{BMO}_{\mathcal{S}}^{\circ}$. Similarly the spaces $\mathrm{BMO}_{\mathcal{P}}^{\circ}$ and $\mathrm{bmo}_{\mathcal{P}}^{\circ}$ embed contractively into $L_{1}(\mathcal{M})$. The same statements 
hold for the completely bounded norms by considerig matrix amplifications. Let $X$ be any of these spaces. We denote the embedding of the complex interpolation spaces by

$$
\kappa_{[X, p ; q]}^{\varphi}:\left[X, L_{p}^{\circ}(\mathcal{M})\right]_{1 / q}^{\varphi} \rightarrow L_{1}^{\circ}(\mathcal{M}) .
$$

Lemma 4.3. Let $\mathcal{M}_{1}$ be a von Neumann subalgebra of $\mathcal{M}$ that is invariant under the semi-group $\mathcal{S}$ and which admits a $\varphi$-preserving conditional expectation $\mathcal{E}$. Then we have 1-complemented inclusions

$$
\operatorname{BMO}_{\mathcal{S}}^{\circ}\left(\mathcal{M}_{1}\right) \subseteq \operatorname{BMO}_{\mathcal{S}}^{\circ}(\mathcal{M}), \quad \operatorname{BMO}_{\mathcal{P}}^{\circ}\left(\mathcal{M}_{1}\right) \subseteq \operatorname{BMO}_{\mathcal{P}}^{\circ}(\mathcal{M})
$$

Moreover, we have a 1-complemented inclusion $\operatorname{bmo}_{\mathcal{P}}^{\circ}\left(\mathcal{M}_{1}\right) \subseteq \operatorname{bmo}_{\mathcal{P}}^{\circ}(\mathcal{M})$ and if $\mathcal{S}$ admits a standard Markov dilation we have a 1-complemented inclusion $\operatorname{bmo}_{\widetilde{\mathcal{P}}}^{\circ}\left(\mathcal{R}_{n}\right) \subseteq \operatorname{bmo}_{\widetilde{\mathcal{P}}}^{\circ}(\mathcal{R})$.

Proof. It is immediate that (4.1) are isometric inclusions. Also for any $t \geq 0$ by the KadisonSchwarz inequality,

$$
\begin{aligned}
& \left\|\Phi_{t}\left(\left|\mathcal{E}(x)-\Phi_{t}(\mathcal{E}(x))\right|^{2}\right)\right\|^{2}=\left\|\Phi_{t}\left(\mathcal{E}\left(x-\Phi_{t}(x)\right)^{*} \mathcal{E}\left(x-\Phi_{t}(x)\right)\right)\right\|^{2} \\
\leq & \left\|\Phi_{t}\left(\mathcal{E}\left(\left(x-\Phi_{t}(x)\right)^{*}\left(x-\Phi_{t}(x)\right)\right)\right)\right\|^{2} \leq\left\|\Phi_{t}\left(\left(x-\Phi_{t}(x)\right)^{*}\left(x-\Phi_{t}(x)\right)\right)\right\|^{2} .
\end{aligned}
$$

Taking the supremum over $t \geq 0$ we see that $\|\mathcal{E}(x)\|_{\mathrm{BMO}_{\mathcal{S}}^{\circ}\left(\mathcal{M}_{1}\right)} \leq\|x\|_{\mathrm{BMO}_{S}^{\circ}(\mathcal{M})}$. The same argument applies to the Poisson semi-group $\mathcal{P}$ so that (4.1) follows. Accoding to [Ana06] a standard Markov dilation for $\mathcal{S}$ yields a Markov dilation for $\mathcal{P}$. The proof of the remaining statements are then similar to Lemmas 3.11 and 3.12 .

The proof of the following proposition is similar to the one of Proposition 4.2.

Proposition 4.4. Let $\mathcal{S}$ be a $\varphi$-modular semi-group. Let $X$ be any of the spaces $\mathrm{BMO}_{\widetilde{\mathcal{S}}}^{\circ}, \mathrm{bmo}_{\widetilde{\mathcal{P}}}^{\circ}$ or $\mathrm{BMO}_{\widetilde{\mathcal{P}}}^{\circ}$. We have a complete isometry

$$
\sigma_{X, p, q, n}:\left[X, L_{p}^{\circ}(\mathcal{R})\right]_{1 / q}^{\widetilde{\varphi}} \rightarrow\left[X, L_{p}^{\circ}(\mathcal{R})\right]_{1 / q}^{\widetilde{\varphi}_{n}}
$$

Moreover, the isometry is explicitly given by

$$
\kappa_{[X, p ; q]}^{\widetilde{\varphi}_{n}} \circ \sigma_{X, p, q, n}(y)=h_{n}^{\frac{1}{2}-\frac{1}{2 p q}} \kappa_{[X, p ; q]}^{\widetilde{\varphi}}(y) h_{n}^{\frac{1}{2}-\frac{1}{2 p q}} .
$$

We now get the following theorem. The KMS-symmetry is only needed because Theorem 4.2 assumes it.

Theorem 4.5. Following the notation introduced above. Assume moreover that $\mathcal{S}$ is a $\varphi$-modular KMS-symmetric Markov semi-group that admits a standard Markov dilation. Then, for all $1 \leq$ $p<\infty, 1<q<\infty$,

$$
\left[X, L_{p}^{\circ}(\mathcal{M})\right]_{1 / q} \approx_{p q} L_{p q}^{\circ}(\mathcal{M})
$$

where $X$ is any of the spaces $\mathrm{BMO}_{\mathcal{S}}^{\circ}$, $\mathrm{bmo}_{\mathcal{P}}^{\circ}$ or $\mathrm{BMO}_{\mathcal{P}}^{\circ}$.

Proof. We sketch the proof. First we observe that again $\mathcal{S}$ may be extended to a Markov semigroup $\widetilde{\mathcal{S}}$ on $\mathcal{R}$ which has a standard Markov dilation, c.f. Proposition 3.3. Again $\widetilde{\mathcal{S}}$ restricts to $\mathcal{R}_{n}$ as a Markov semi-group with respect to $\widetilde{\varphi}_{n}$. Depending on which space $X$ is (as in the statement of the theorem) we define the following. Let $Y$ be either $\operatorname{BMO}_{\mathcal{S}}^{\circ}(\mathcal{R}), \operatorname{bmo}_{\mathcal{P}}^{\circ}(\mathcal{R})$ or $\operatorname{BMO}_{\mathcal{P}}^{\circ}(\mathcal{R})$. Let $Y_{n}$ be either $\mathrm{BMO}_{\mathcal{S}}^{\circ}\left(\mathcal{R}_{n}\right)$, bmo $_{\mathcal{P}}^{\circ}\left(\mathcal{R}_{n}\right)$ or $\mathrm{BMO}_{\mathcal{P}}^{\circ}\left(\mathcal{R}_{n}\right)$. We may therefore apply the tracial Theorem 
4.2 to interpolate for each $n$ and find $\left[Y_{n}, L_{p}^{\circ}\left(\mathcal{R}_{n}\right)\right]_{1 / q}^{\widetilde{\varphi_{n}}} \approx_{p q} L_{p q}^{\circ}\left(\mathcal{R}_{n}\right)$. One now checks that there is a diagram

$$
\begin{array}{rrr}
{\left[Y_{n}, L_{p}^{\circ}\left(\mathcal{R}_{n}\right)\right]_{1 / q}^{\widetilde{\varphi}_{n}}} & \stackrel{\subseteq}{\longrightarrow}\left[Y, L_{p}^{\circ}(\mathcal{R})\right]_{1 / q}^{\widetilde{\varphi}_{n}} \\
\approx_{p q} \uparrow & \begin{array}{r}
\sigma_{X, p, q, n}^{-1} \\
L_{p q}^{\circ}\left(\mathcal{R}_{n}\right)
\end{array} \\
& {\left[Y, L_{p}^{\circ}(\mathcal{R})\right]_{1 / q}^{\widetilde{\varphi}} .}
\end{array}
$$

that is compatible with respect to the interpolation structure of $\widetilde{\varphi}$. The remainder of the proof is then exactly the same as in Theorem 3.15.

\section{Fourier multipliers on free Araki-Woods factors}

We recall the definiton of free Araki-Woods factors from [Shl97]. Let $\mathcal{H}_{\mathbb{R}}$ be a real Hilbert space and let $\mathcal{H}_{\mathbb{C}}=\mathcal{H}_{\mathbb{R}} \otimes_{\mathbb{R}} \mathbb{C}$ be its complexification. For $\xi \in \mathcal{H}_{\mathbb{C}}$ with $\xi=\xi_{1}+i \xi_{2}$ and $\xi_{1}, \xi_{2} \in \mathcal{H}_{\mathbb{R}}$ we set $\bar{\xi}=\xi_{1}-i \xi_{2}$. Let $\left(V_{t}\right)_{t \in \mathbb{R}}$ be a strongly continuous 1-parameter group of orthogonal transformations on $\mathcal{H}_{\mathbb{R}}$ and use the same notation for its extension to a strongly continuous unitary 1-parameter group on $\mathcal{H}_{\mathbb{C}}$. Through Stone's theorem we have $V_{t}=A^{i t}$ where $A$ is a positive (possibly) unbounded self-adjoint operator on $\mathcal{H}_{\mathbb{C}}$. We define a new innerproduct on $\mathcal{H}_{\mathbb{C}}$ by setting $\langle\xi, \eta\rangle_{A}=\left\langle\frac{2 A}{1+A} \xi, \eta\right\rangle$. Let $\mathcal{H}$ be the completion of $\mathcal{H}_{\mathbb{C}}$ with respect to the latter inner product. We have that the embedding $\mathcal{H}_{\mathbb{R}} \hookrightarrow \mathcal{H}$ is isometric [Shl97, p. 332]. We construct a Fock space,

$$
\mathcal{F}=\mathbb{C} \Omega \oplus \bigoplus_{k=1}^{\infty} \mathcal{H}^{\otimes k}
$$

We denote $\varphi_{\Omega}$ for the vector state $x \mapsto\langle x \Omega, \Omega\rangle$. For $\xi \in \mathcal{H}$ let $a(\xi)$ be the creation operator on $\mathcal{F}$ defined by

$$
a(\xi): \eta_{1} \otimes \ldots \otimes \eta_{k} \mapsto \xi \otimes \eta_{1} \otimes \ldots \otimes \eta_{k} .
$$

Let $a^{*}(\xi)$ be its adjoint which is the annihilation operator

$$
a^{*}(\xi): \eta_{1} \otimes \ldots \otimes \eta_{k} \mapsto\left\langle\eta_{1}, \xi\right\rangle_{A} \eta_{2} \otimes \ldots \otimes \eta_{k} .
$$

For $\xi \in \mathcal{H}$ define the self-adjoint operator $s(\xi)=a(\xi)+a^{*}(\xi)$. Let,

$$
\mathcal{M}:=\mathcal{A}_{0}^{\prime \prime} \text { with } \mathcal{A}_{0}:=\Gamma\left(\mathcal{H}_{\mathbb{R}},\left(V_{t}\right)_{t}\right):=\left\langle s(\xi) \mid \xi \in \mathcal{H}_{\mathbb{R}}\right\rangle,
$$

where $\left\langle s(\xi) \mid \xi \in \mathcal{H}_{\mathbb{R}}\right\rangle$ stands for the $*$-algebra generated by these operators. The von Neumann algebra $\mathcal{M}$ is called the free Araki-Woods algebra. The vacuüm vector $\Omega$ is separating and cyclic for this algebra. Set $\varphi_{\Omega}(\cdot)=\langle\cdot \Omega, \Omega\rangle$. Therefore if for $\xi \in \mathcal{F}$ there is an operator $W(\xi)$ such that $W(\xi) \Omega=\xi$, then this operator is unique. For various calculations and to define suitable Fourier multipliers in the first place we need the following Wick theorem.

Theorem 5.1 (See Proposition 2.7 of [BKS07] or Lemma 3.2 of [HoRi11]). Suppose that $\xi_{1}, \ldots, \xi_{n} \in$ $\mathcal{H}_{\mathbb{C}}$ then,

$$
W\left(\xi_{1} \otimes \ldots \otimes \xi_{n}\right)=\sum_{j=0}^{n} a\left(\xi_{1}\right) \ldots a\left(\xi_{j}\right) a^{*}\left(\overline{\xi_{j+1}}\right) \ldots a^{*}\left(\overline{\xi_{n}}\right) .
$$

The linear span of operators of the form (5.1) form a $*$-algebra which we shall denote by $\mathcal{A}$ (in fact, this follows from (5.5) below). Moreover $\mathcal{A}$ is dense in $\mathcal{M}$. 
If $T$ is a contractive operator on $\mathcal{H}_{\mathbb{R}}$ such that for every $t \in \mathbb{R}$ we have $T V_{t}=V_{t} T$ then there exists a unique normal ucp map (see [Hia01], [Was17, Proposition 3.3] for the even more general result for the $q$-Araki-Woods case),

$$
\Gamma(T): \mathcal{M} \rightarrow \mathcal{M}: W\left(\xi_{1} \otimes \ldots \otimes \xi_{n}\right) \mapsto W\left(T \xi_{1} \otimes \ldots \otimes T \xi_{n}\right) .
$$

This assignment is called second quantization. We are now ready to define the Hilbert transform.

Definition 5.2. Fix spaces $\mathcal{H}_{\mathbb{C}}^{ \pm} \subseteq \mathcal{H}_{\mathbb{C}}$ that are closed in $\mathcal{H}_{\mathbb{C}}$ and such that $\mathcal{H}_{\mathbb{C}}^{+} \cap \mathcal{H}_{\mathbb{C}}^{-}=\{0\}$. So as Banach spaces $\mathcal{H}_{\mathbb{C}}=\mathcal{H}_{\mathbb{C}}^{+} \oplus \mathcal{H}_{\mathbb{C}}^{-}$. Assume moreover that $\mathcal{H}_{\mathbb{C}}^{+}$and $\mathcal{H}_{\mathbb{C}}^{-}$are orthogonal in $\mathcal{H}$ for the inner product $\langle,\rangle_{A}$. Set $\epsilon=\left(\mathcal{H}_{\mathbb{C}}^{+}, \mathcal{H}_{\mathbb{C}}^{-}\right)$. The mapping $H_{\epsilon}: \mathcal{A} \rightarrow \mathcal{A}$ defined as the linear extension of

$$
H_{\epsilon}: W\left(\xi_{1} \otimes \ldots \otimes \xi_{n}\right)= \pm W\left(\xi_{1} \otimes \ldots \otimes \xi_{n}\right),
$$

with $\xi_{1} \in \mathcal{H}_{\mathbb{C}}^{ \pm}, \xi_{2}, \ldots, \xi_{n} \in \mathcal{H}_{\mathbb{C}}$ and $H_{\epsilon}(1)=0$ will be called the Hilbert transform (which only depends on the decomposition $\mathcal{H}_{\mathbb{C}}=\mathcal{H}_{\mathbb{C}}^{+} \oplus \mathcal{H}_{\mathbb{C}}^{-}$.

Remark 5.3. Let $\left(\sigma_{t}\right)_{t \in \mathbb{R}}$ be the modular automorphism group of $\varphi_{\Omega}$. We have

$$
\sigma_{t}\left(W\left(\xi_{1} \otimes \ldots \otimes \xi_{n}\right)\right)=W\left(\left(A^{i t} \xi_{1}\right) \otimes \ldots \otimes\left(A^{i t} \xi_{n}\right)\right),
$$

see [Sh197]. Suppose that the spaces $\mathcal{H}_{\mathbb{C}}^{ \pm}$are invariant subspaces for all $A^{i t}, t \in \mathbb{R}$. It follows that $\sigma_{t}$ and $H_{\epsilon}$ with $\epsilon=\left(\mathcal{H}_{\mathbb{C}}^{+}, \mathcal{H}_{\mathbb{C}}^{-}\right)$commute for all $t \in \mathbb{R}$.

5.1. $L^{p}$-boundedness and Cotlar's trick. To define a fixed Hilbert transform we prefix a decomposition $\mathcal{H}_{\mathbb{C}}^{+} \oplus \mathcal{H}_{\mathbb{C}}^{-}$of the Hilbert space $\mathcal{H}_{\mathbb{C}}$. Here the $\mathcal{H}_{\mathbb{C}}^{ \pm}$are closed in $\mathcal{H}_{\mathbb{C}}$ and orthogonal in $\mathcal{H}$. Set $\epsilon=\left(\mathcal{H}_{\mathbb{C}}^{+}, \mathcal{H}_{\mathbb{C}}^{-}\right)$as before. We write $\mathcal{E}_{\Omega}^{\perp}(x)=x-\varphi_{\Omega}(x)$. This is the orthocomplement of the projection onto $\mathbb{C} 1 \subseteq \mathcal{M}$ with respect to the inner product of the vacuüm state.

Proposition 5.4 (Cotlar formula for the Hilbert transform). The following relation holds true:

$$
\mathcal{E}_{\Omega}^{\perp}\left(H_{\epsilon}(x) H_{\epsilon}(y)^{*}\right)=\mathcal{E}_{\Omega}^{\perp}\left(H_{\epsilon}\left(x H_{\epsilon}(y)^{*}\right)+H_{\epsilon}\left(y H_{\epsilon}(x)^{*}\right)^{*}-H_{\epsilon}\left(H_{\epsilon}\left(x y^{*}\right)^{*}\right)^{*}\right),
$$

for all $x, y \in \mathcal{A}$.

Proof. By linearity we may assume that $x$ and $y$ are Wick operators of elementary tensors. So say $x=W\left(\xi_{1} \otimes \ldots \otimes \xi_{m}\right)$ and $y=W\left(\eta_{1} \otimes \ldots \otimes \eta_{n}\right)$. Moreover, assume that $\xi_{1} \in \mathcal{H}_{\mathbb{C}}^{\epsilon_{x}}, \eta_{1} \in \mathcal{H}_{\mathbb{C}}^{\epsilon_{y}}$ for signs $\epsilon_{x}, \epsilon_{y}= \pm 1$. By (5.1) we get,

$$
\begin{aligned}
& x y^{*}= \\
& \left(\sum_{r=0}^{m} a\left(\xi_{1}\right) \ldots a\left(\xi_{r}\right) a^{*}\left(\overline{\xi_{r+1}}\right) \ldots a^{*}\left(\overline{\xi_{m}}\right)\right)\left(\sum_{s=0}^{n} a\left(\overline{\eta_{n}}\right) \ldots a\left(\overline{\eta_{s+1}}\right) a^{*}\left(\eta_{s}\right) \ldots a^{*}\left(\eta_{1}\right) .\right)
\end{aligned}
$$

We rename vectors by setting $\left(\mu_{1}, \ldots, \mu_{n+m}\right)=\left(\xi_{1}, \ldots, \xi_{m}, \overline{\eta_{n}}, \ldots, \overline{\eta_{1}}\right)$. In the first equality in the next computation we collect the terms in (5.4) by separating the ones where no annihilation operator is on the left of a creation operator (first summand of (5.5)) and the ones where such a combination does occur (second summand of (5.5)). The second equation is the Wick formula 
$(5.1)$,

$$
\begin{aligned}
x y^{*}= & \sum_{r=0}^{n+m} a\left(\mu_{1}\right) \ldots a\left(\mu_{r}\right) a^{*}\left(\overline{\mu_{r+1}}\right) \ldots a^{*}\left(\overline{\mu_{n+m}}\right) \\
& +\left(\sum_{r=0}^{m-1} a\left(\xi_{1}\right) \ldots a\left(\xi_{r}\right) a^{*}\left(\overline{\xi_{r+1}}\right) \ldots a^{*}\left(\overline{\xi_{m-1}}\right)\right) a^{*}\left(\overline{\xi_{m}}\right) a\left(\overline{\eta_{n}}\right) \\
& \times \quad\left(\sum_{s=0}^{n-1} a\left(\overline{\eta_{n-1}}\right) \ldots a\left(\overline{\eta_{s+1}}\right) a^{*}\left(\eta_{s}\right) \ldots a^{*}\left(\eta_{1}\right)\right) \\
= & W\left(\xi_{1} \otimes \ldots \otimes \xi_{m} \otimes \overline{\eta_{n}} \otimes \ldots \otimes \overline{\eta_{1}}\right) \\
& +\left\langle\overline{\eta_{n}}, \overline{\xi_{m}}\right\rangle_{A} W\left(\xi_{1} \otimes \ldots \otimes \xi_{m-1}\right) W\left(\eta_{1} \otimes \ldots \otimes \eta_{n-1}\right)^{*} .
\end{aligned}
$$

Now we separate cases. Note that we may assume that $n, m \neq 0$ because otherwise the proposition is trivial.

Case 1: Assume $m>n>0$. Applying the equation (5.5) inductively on the length of $m$ we see that,

$$
x y^{*}=\sum_{k=0}^{n} \prod_{l=0}^{k-1}\left\langle\overline{\eta_{n-l}}, \overline{\xi_{m-l}}\right\rangle_{A} W\left(\xi_{1} \otimes \ldots \otimes \xi_{m-k} \otimes \overline{\eta_{n-k}} \otimes \ldots \otimes \overline{\eta_{1}}\right) .
$$

Here the $k=0$ term is understood as $W\left(\xi_{1} \otimes \ldots \otimes \xi_{m} \otimes \overline{\eta_{n}} \otimes \ldots \otimes \overline{\eta_{1}}\right)$. In particular as $m>n$ we see that $\mathcal{E}_{\Omega}^{\perp}\left(x y^{*}\right)=x y^{*}$ and similarly

$$
\begin{aligned}
\mathcal{E}_{\Omega}^{\perp}\left(H_{\epsilon}\left(x H_{\epsilon}(y)^{*}\right)\right) & =H_{\epsilon}\left(x H_{\epsilon}(y)^{*}\right) \\
\mathcal{E}_{\Omega}^{\perp}\left(H_{\epsilon}\left(y H_{\epsilon}(x)^{*}\right)^{*}\right) & =H_{\epsilon}\left(y H_{\epsilon}(x)^{*}\right)^{*} \\
\mathcal{E}_{\Omega}^{\perp}\left(H_{\epsilon}\left(H_{\epsilon}\left(x y^{*}\right)^{*}\right)^{*}\right) & =H_{\epsilon}\left(H_{\epsilon}\left(x y^{*}\right)^{*}\right)^{*}
\end{aligned}
$$

So to prove the Cotlar identity (5.3) we can ignore the projection $\mathcal{E}_{\Omega}^{\perp}$ in this case. Now for the right hand side of the Cotlar identity (5.3) we argue that we get the Equation (5.7) below. Firstly, because $H_{\epsilon}(y)=\epsilon_{y} y$ we find that $x H_{\epsilon}(y)^{*}$ equals $\epsilon_{y}$ times the expression (5.6). Then, as $m>n$,

$$
H_{\epsilon}\left(x H_{\epsilon}(y)^{*}\right)=\sum_{k=0}^{n} \epsilon_{y} \epsilon_{x} \prod_{l=0}^{k-1}\left\langle\overline{\eta_{n-l}}, \overline{\xi_{m-l}}\right\rangle_{A} W\left(\xi_{1} \otimes \ldots \otimes \xi_{m-k} \otimes \overline{\eta_{n-k}} \otimes \ldots \otimes \overline{\eta_{1}}\right) .
$$

Secondly, $y H_{\epsilon}(x)^{*}=\epsilon_{x} y x^{*}$. Then, $y H_{\epsilon}(x)^{*}$ is $\epsilon_{x}$ times the adjoint of the expression (5.6). Then, we get the following two summands, where the second line appears as if $k=n$ then there is no more tensor $\eta_{1}$ appearing in the decomposition of $y H_{\epsilon}(x)^{*}$ in terms of Wick words,

$$
\begin{aligned}
H_{\epsilon}\left(y H_{\epsilon}(x)^{*}\right)= & \sum_{k=0}^{n-1} \epsilon_{x} \epsilon_{y} \prod_{l=0}^{k-1}\left\langle\overline{\xi_{m-l}}, \overline{\eta_{n-l}}\right\rangle_{A} W\left(\eta_{1} \otimes \ldots \otimes \eta_{n-k} \otimes \overline{\xi_{m-k}} \otimes \ldots \otimes \overline{\xi_{1}}\right) \\
& +\epsilon_{x} \prod_{l=0}^{n-1}\left\langle\overline{\xi_{m-l}}, \overline{\eta_{n-l}}\right\rangle_{A} H_{\epsilon}\left(W\left(\overline{\xi_{m-n}} \otimes \ldots \otimes \overline{\xi_{1}}\right)\right) .
\end{aligned}
$$


By a similar argument we get also that,

$$
\begin{aligned}
H_{\epsilon}\left(H_{\epsilon}\left(x y^{*}\right)^{*}\right)= & \sum_{k=0}^{n-1} \epsilon_{x} \epsilon_{y} \prod_{l=0}^{k-1}\left\langle\overline{\xi_{m-l}}, \overline{\eta_{n-l}}\right\rangle_{A} W\left(\eta_{1} \otimes \ldots \otimes \eta_{n-k} \otimes \overline{\xi_{m-k}} \otimes \ldots \otimes \overline{\xi_{1}}\right) \\
& +\epsilon_{x} \prod_{l=0}^{n-1}\left\langle\overline{\xi_{m-l}}, \overline{\eta_{n-l}}\right\rangle_{A} H_{\epsilon}\left(W\left(\overline{\xi_{m-n}} \otimes \ldots \otimes \overline{\xi_{1}}\right)\right) .
\end{aligned}
$$

Then,

$$
\begin{aligned}
& H_{\epsilon}\left(x H_{\epsilon}(y)^{*}\right)+H_{\epsilon}\left(y H_{\epsilon}(x)^{*}\right)^{*}-H_{\epsilon}\left(H_{\epsilon}\left(x y^{*}\right)^{*}\right)^{*} \\
= & \epsilon_{x} \epsilon_{y} \sum_{k=0}^{n} \prod_{l=0}^{k-1}\left\langle\overline{\eta_{n-l}}, \overline{\xi_{m-l}}\right\rangle_{A} W\left(\xi_{1} \otimes \ldots \otimes \xi_{m-k} \otimes \overline{\eta_{n-k}} \otimes \ldots \otimes \overline{\eta_{1}}\right) .
\end{aligned}
$$

On the other hand, from (5.4) we conclude that,

$$
H_{\epsilon}(x) H_{\epsilon}(y)^{*}=\epsilon_{x} \epsilon_{y} x y^{*},
$$

which equals (5.7) by (5.5).

Case 2: Assume $m=n>0$. Because as $H_{\epsilon}(1)=0$ we find the following decomposition (so the summand $k=n$ vanishes),

$$
H_{\epsilon}\left(x H_{\epsilon}(y)^{*}\right)=\sum_{k=0}^{n-1} \epsilon_{y} \epsilon_{x} \prod_{l=0}^{k-1}\left\langle\overline{\eta_{n-l}}, \overline{\xi_{m-l}}\right\rangle_{A} W\left(\xi_{1} \otimes \ldots \otimes \xi_{m-k} \otimes \overline{\eta_{n-k}} \otimes \ldots \otimes \overline{\eta_{1}}\right) .
$$

Further, again as as $H_{\epsilon}(1)=0$,

$$
H_{\epsilon}\left(y H_{\epsilon}(x)^{*}\right)=\sum_{k=0}^{n-1} \epsilon_{x} \epsilon_{y} \prod_{l=0}^{k-1}\left\langle\overline{\eta_{n-l}}, \overline{\xi_{m-l}}\right\rangle_{A} W\left(\eta_{1} \otimes \ldots \otimes \eta_{n-k} \otimes \overline{\xi_{m-k}} \otimes \ldots \otimes \overline{\eta_{1}}\right),
$$

and

$$
H_{\epsilon}\left(H_{\epsilon}\left(x y^{*}\right)^{*}\right)=\sum_{k=0}^{n-1} \epsilon_{x} \epsilon_{y} \prod_{l=0}^{k-1}\left\langle\overline{\eta_{n-l}}, \overline{\xi_{m-l}}\right\rangle_{A} W\left(\eta_{1} \otimes \ldots \otimes \eta_{n-k} \otimes \overline{\xi_{m-k}} \otimes \ldots \otimes \overline{\xi_{1}}\right) .
$$

Then,

$$
\begin{aligned}
& H_{\epsilon}\left(x H_{\epsilon}(y)^{*}\right)+H_{\epsilon}\left(y H_{\epsilon}(x)^{*}\right)^{*}-H_{\epsilon}\left(H_{\epsilon}\left(x y^{*}\right)^{*}\right)^{*} \\
= & \epsilon_{x} \epsilon_{y} \sum_{k=0}^{n-1} \prod_{l=0}^{k-1}\left\langle\overline{\eta_{n-l}}, \overline{\xi_{m-l}}\right\rangle_{A} W\left(\xi_{1} \otimes \ldots \otimes \xi_{m-k} \otimes \overline{\eta_{n-k}} \otimes \ldots \otimes \overline{\eta_{1}}\right) .
\end{aligned}
$$

This expression is in the range of the projection $\mathcal{E}_{\Omega}^{\perp}$. On the other hand, by (5.5) and using $n=m$ we get

$$
\begin{aligned}
& \mathcal{E}_{\Omega}^{\perp}\left(H_{\epsilon}(x) H_{\epsilon}(y)^{*}\right)=\epsilon_{x} \epsilon_{y} \mathcal{E}_{\Omega}^{\perp}\left(x y^{*}\right) \\
= & \epsilon_{x} \epsilon_{y} \sum_{k=0}^{n-1} \prod_{l=0}^{k-1}\left\langle\overline{\eta_{n-l}}, \overline{\xi_{m-l}}\right\rangle_{A} W\left(\xi_{1} \otimes \ldots \otimes \xi_{m-k} \otimes \overline{\eta_{n-k}} \otimes \ldots \otimes \overline{\eta_{1}}\right),
\end{aligned}
$$

which concludes the proof of Case 2 as this equals (5.9). 
Case 3: Assume $n>m$. The proof can be obtained by a mutatis mutandis copy of Case 1 . We sketch a second way to finish the proof. By Case 1:

$$
H_{\epsilon}(y) H_{\epsilon}(x)^{*}=H_{\epsilon}\left(y H_{\epsilon}(x)^{*}\right)+H_{\epsilon}\left(x H_{\epsilon}(y)^{*}\right)^{*}-H_{\epsilon}\left(H_{\epsilon}\left(y x^{*}\right)^{*}\right)^{*} .
$$

Then one verifies that $H_{\epsilon}\left(H_{\epsilon}\left(y x^{*}\right)^{*}\right)=H_{\epsilon}\left(H_{\epsilon}\left(x y^{*}\right)^{*}\right)^{*}$. So that taking adjoints of (5.11) we see,

$$
H_{\epsilon}(x) H_{\epsilon}(y)^{*}=H_{\epsilon}\left(y H_{\epsilon}(x)^{*}\right)^{*}+H_{\epsilon}\left(x H_{\epsilon}(y)^{*}\right)-H_{\epsilon}\left(H_{\epsilon}\left(x y^{*}\right)^{*}\right)^{*} .
$$

We write $D$ for the operator $D_{\varphi_{\Omega}}$.

Lemma 5.5. For $x \in \mathcal{A}$ we have that

$$
\varphi_{\Omega}\left(H_{\epsilon}(x)^{*} H_{\epsilon}(x)\right)=\varphi_{\Omega}\left(x^{*} x\right) .
$$

So certainly for every $1 \leq p<\infty$ we get that $\left\|D^{\frac{1}{2 p}} \varphi_{\Omega}\left(H_{\epsilon}(x)^{*} H_{\epsilon}(x)\right) D^{\frac{1}{2 p}}\right\|_{p}=\left\|D^{\frac{1}{2 p}} \varphi_{\Omega}\left(x^{*} x\right) D^{\frac{1}{2 p}}\right\|_{p}$.

Proof. As $x$ is in the algebra $\mathcal{A}$ we may take a decomposition $x=x^{+}+x^{-}$with $x^{ \pm}$in the linear span of Wick operators $W\left(\xi_{1} \otimes \ldots \otimes \xi_{n}\right), \xi_{1} \in \mathcal{H}_{\mathbb{C}}^{ \pm}$. We have

$$
\varphi_{\Omega}\left(H_{\epsilon}(x)^{*} H_{\epsilon}(x)\right)=\left\langle x^{+} \Omega, x^{+} \Omega\right\rangle+\left\langle x^{-} \Omega, x^{-} \Omega\right\rangle-\left\langle x^{+} \Omega, x^{-} \Omega\right\rangle-\left\langle x^{-} \Omega, x^{+} \Omega\right\rangle .
$$

As $\mathcal{H}_{\mathbb{C}}^{+}$and $\mathcal{H}_{\mathbb{C}}^{-}$are orthogonal for the inner product of $\mathcal{H}$ we find that

$$
\varphi_{\Omega}\left(H_{\epsilon}(x)^{*} H_{\epsilon}(x)\right)=\left\langle x^{+} \Omega, x^{+} \Omega\right\rangle+\left\langle x^{-} \Omega, x^{-} \Omega\right\rangle=\varphi_{\Omega}\left(x^{*} x\right) .
$$

Theorem 5.6. For every $1<p<\infty$ and every choice of $\epsilon=\left(\mathcal{H}_{\mathbb{C}}^{+}, \mathcal{H}_{\mathbb{C}}^{-}\right)$as in Definition 5.2 such that $A^{\text {it }}$ leaves $\mathcal{H}_{\mathbb{C}}^{ \pm}$invariant for all $t \in \mathbb{R}$ the map $H_{\epsilon}$ extends to a bounded map $L_{p}(\mathcal{M}) \rightarrow L_{p}(\mathcal{M})$ that is determined by

$$
H_{\epsilon}: D^{\frac{1}{2 p}} x D^{\frac{1}{2 p}} \mapsto D^{\frac{1}{2 p}} H_{\epsilon}(x) D^{\frac{1}{2 p}}, \quad x \in \mathcal{A}
$$

Moreover, let $c_{p}$ be the norm of (5.12). Then for $p \geq 2$ a power of 2 we have $c_{p} \leq p^{\gamma / 2}$ with $\gamma={ }^{3} \log (2)$. Further, for $C=2^{\gamma / 2}$ we have $c_{p} \leq C p^{\gamma / 2}$ for $p \geq 2$ arbitrary.

Proof. For $p=2$ the map $H_{\epsilon}$ defines a contraction on $L_{2}(\mathcal{M})$ and so the statement is true.

The space $D^{\frac{1}{2 p}} \mathcal{A} D^{\frac{1}{2 p}}$ is dense in $L_{p}(\mathcal{M})$. As $H_{\epsilon}: \mathcal{A} \rightarrow \mathcal{A}$ commutes with the modular automorphism group of $\varphi_{\Omega}$, c.f. Remark 5.3, it follows from a computation similar to (2.2) that,

$$
H_{\epsilon}\left(D^{\frac{1}{p}} x\right)=D^{\frac{1}{p}} H_{\epsilon}(x) .
$$


We now estimate $c_{2 p}$ in terms of $c_{p}$. By Cotlar's identity (5.3) and Lemma 5.5 we get that,

$$
\begin{aligned}
& \left\|D^{\frac{1}{2 p}} H_{\epsilon}(x)\right\|_{2 p}^{2} \\
= & \left\|D^{\frac{1}{2 p}} H_{\epsilon}(x) H_{\epsilon}(x)^{*} D^{\frac{1}{2 p}}\right\|_{p} \\
\leq & \left\|D^{\frac{1}{2 p}} \mathcal{E}_{\Omega}^{\perp}\left(H_{\epsilon}(x) H_{\epsilon}(x)^{*}\right) D^{\frac{1}{2 p}}\right\|_{p}+\left\|D^{\frac{1}{2 p}} \varphi_{\Omega}\left(H_{\epsilon}(x) H_{\epsilon}(x)^{*}\right) D^{\frac{1}{2 p}}\right\|_{p} \\
\leq & \left\|D^{\frac{1}{2 p}} H_{\epsilon}\left(x H_{\epsilon}(x)^{*}\right) D^{\frac{1}{2 p}}\right\|_{p}+\left\|D^{\frac{1}{2 p}} H_{\epsilon}\left(x H_{\epsilon}(x)^{*}\right)^{*} D^{\frac{1}{2 p}}\right\|_{p} \\
& \quad\left\|D^{\frac{1}{2 p}} H_{\epsilon}\left(H_{\epsilon}\left(x x^{*}\right)^{*}\right)^{*} D^{\frac{1}{2 p}}\right\|_{p}+\left\|D^{\frac{1}{2 p}} \varphi_{\Omega}\left(x x^{*}\right) D^{\frac{1}{2 p}}\right\|_{2 p} \\
\leq & c_{p}\left\|D^{\frac{1}{2 p}} x\right\|_{2 p}\left\|H_{\epsilon}(x)^{*} D^{\frac{1}{2 p}}\right\|_{2 p}+c_{p}\left\|D^{\frac{1}{2 p}} x\right\|_{2 p}\left\|H_{\epsilon}(x)^{*} D^{\frac{1}{2 p}}\right\|_{2 p} \\
& \quad+c_{p}^{2}\left\|D^{\frac{1}{2 p}} x\right\|_{2 p}^{2}+\left\|D^{\frac{1}{2 p}} x\right\|_{2 p}^{2} \\
= & 2 c_{p}\left\|D^{\frac{1}{2 p}} x\right\|_{2 p}\left\|H_{\epsilon}(x)^{*} D^{\frac{1}{2 p}}\right\|_{2 p}+\left(c_{p}^{2}+1\right)\left\|D^{\frac{1}{2 p}} x\right\|_{2 p}^{2} .
\end{aligned}
$$

By density we conclude that $c_{2 p} \leq c_{p}+\sqrt{2 c_{p}^{2}+1}$. In particular $c_{2 p} \leq(1+\sqrt{2}) c_{p}$ from which it follows that for $p$ a power of 2 we get that $c_{p} \leq p^{\gamma}$ with $\gamma=\frac{\log (2)}{\log (1+\sqrt{3})}$. For other $p \geq 2$ the result follows by interpolation, see [BeLö76], [Ter82].

Remark 5.7. We do not know what the optimal constants are for the norm of $H_{\epsilon}$ on $L_{p}(\mathcal{M})$. We also leave it as an open question whether the Hilbert transform is a bounded map $L_{\infty} \rightarrow$ BMO or even $\mathrm{BMO} \rightarrow \mathrm{BMO}$ as for the classical Hilbert transform.

5.2. Khintchine type BMO inequalities and multipliers. We provide examples of $L_{\infty} \rightarrow$ BMO-multipliers on free Araki-Woods factors. Earlier results on non-commutative $L_{\infty} \rightarrow$ BMOmultipliers in the tracial setting were obtained by Mei [Mei17] but here we do not need to appeal to lacunary sets. We use the Markov semi-group $\mathcal{S}=\left(\Psi_{t}=\Phi_{e^{-t}}\right)_{t \geq 0}$ that is determined by

$$
\Phi_{r}: W\left(\xi_{1} \otimes \ldots \otimes \xi_{n}\right) \mapsto r^{n} W\left(\xi_{1} \otimes \ldots \otimes \xi_{n}\right), \quad 0 \leq r \leq 1 .
$$

This semi-group is well-known to be Markov, KMS-symmetric and $\varphi_{\Omega}$-modular.

Proposition 5.8. Suppose that $\mathcal{H}$ is infinite dimensional. Let $\left(e_{k}\right)_{k}$ be a set of vectors in $\mathcal{H}_{\mathbb{C}}$ that are orthogonal in $\mathcal{H}$. For $i=\left(i_{1}, \ldots, i_{n}\right)$ a multi-index set $e_{i}=e_{i_{1}} \otimes \ldots \otimes e_{i_{n}}$ and $\bar{e}_{i}=\bar{e}_{i_{1}} \otimes \ldots \otimes \bar{e}_{i_{n}}$. Take $F$ a set of multi-indices such that $\left\langle e_{i_{1}}, e_{j_{1}}\right\rangle_{A}=\left\langle\bar{e}_{i_{1}}, \bar{e}_{j_{1}}\right\rangle_{A}=0$ if $i \neq j$. We have, for any $x=\sum_{i \in F} c_{i} W\left(e_{i}\right)$ with $c_{i} \in \mathbb{C}$ a finite sum of Wick operators whose frequency support lies in $F$, that,

$$
\|x\|_{\mathrm{bmo \mathcal {S }}}^{2} \leq 2 \max \left\{\left\|\sum_{i}\left|c_{i}\right|^{2} W\left(e_{i}\right)^{*} W\left(e_{i}\right)\right\|,\left\|\sum_{i}\left|c_{i}\right|^{2} W\left(e_{i}\right) W\left(e_{i}\right)^{*}\right\|\right\} .
$$

Proof. Using the definition of $x$ and the triangle inequality,

$$
\begin{aligned}
& \left\|\Phi_{r}(x)^{*} \Phi_{r}(x)-\Phi_{r}\left(x^{*} x\right)\right\| \\
\leq & \left\|\sum_{i=j} \overline{c_{i}} c_{j}\left(r^{|i|+|j|}-\Phi_{r}\right)\left(W\left(e_{i}\right)^{*} W\left(e_{j}\right)\right)\right\|+\left\|\sum_{i \neq j} \overline{c_{i}} c_{j}\left(r^{|i|+|j|}-\Phi_{r}\right)\left(W\left(e_{i}\right)^{*} W\left(e_{j}\right)\right)\right\|
\end{aligned}
$$

If $i \neq j$ we get that $\left\langle e_{i_{1}}, e_{j_{1}}\right\rangle_{A}=0$, so that by (5.5) we see that $W\left(e_{i}\right)^{*} W\left(e_{j}\right)=W\left(e_{i}^{*} \otimes e_{j}\right)$ where $e_{i}^{*}=\bar{e}_{i_{n}} \otimes \ldots \otimes \bar{e}_{i_{1}}$ so that,

$$
\left(r^{|i|+|j|}-\Phi_{r}\right)\left(W\left(e_{i}\right)^{*} W\left(e_{j}\right)\right)=\left(r^{|i|+|j|}-r^{|i|+|j|}\right)\left(W\left(e_{i}^{*} \otimes e_{j}\right)\right)=0 .
$$


Therefore we continue (5.15) by using that $\Phi_{r}$ is a ucp map and that the expression $\sum_{i}\left|c_{i}\right|^{2} W\left(e_{i}\right)^{*} W\left(e_{i}\right)$ is a summation of positive elements,

$$
\begin{aligned}
\left\|\Phi_{r}(x)^{*} \Phi_{r}(x)-\Phi_{r}\left(x^{*} x\right)\right\| & =\left\|\sum_{i}\left|c_{i}\right|^{2}\left(r^{2|i|}-\Phi_{r}\right)\left(W\left(e_{i}\right)^{*} W\left(e_{i}\right)\right)\right\| \\
& \leq 2\left\|\sum_{i}\left|c_{i}\right|^{2}\left(W\left(e_{i}\right)^{*} W\left(e_{i}\right)\right)\right\| .
\end{aligned}
$$

This shows that

$$
\|x\|_{\mathrm{bmo}^{c}}^{2} \leq 2\left\|\sum_{i}\left|c_{i}\right|^{2} W\left(e_{i}\right)^{*} W\left(e_{i}\right)\right\|
$$

Then in the same way using the orthogonality $\left\langle\bar{e}_{i_{1}}, \bar{e}_{j_{1}}\right\rangle=0, i \neq j$ we get that $\|x\|_{\mathrm{bmo}^{r}}^{2}=$ $\left\|x^{*}\right\|_{\mathrm{bmo}^{c}}^{2} \leq 2\left\|\sum_{i}\left|c_{i}\right|^{2} W\left(e_{i}\right) W\left(e_{i}\right)^{*}\right\|$.

Let $\delta_{F}$ be the indicator function on a set $F$. The following Corollary 5.9 is a consequence of our interpolation result of Theorem 3.15. We call $n$ the length of a multi-index $i=\left(i_{1}, \ldots, i_{n}\right)$.

Corollary 5.9. Take $F$ a set of multi-indices of length at most $n$ such that $\left\langle e_{i_{1}}, e_{j_{1}}\right\rangle_{A}=\left\langle\bar{e}_{i_{1}}, \bar{e}_{j_{1}}\right\rangle_{A}=$ 0 if $i \neq j$. The projection $P_{F}: W\left(e_{i}\right) \mapsto \delta_{F}(i) W\left(e_{i}\right)$ extends to a bounded map $\mathcal{M} \rightarrow \operatorname{bmos}_{\mathcal{S}}(\mathcal{M})$. Consequently, $P_{F}$ determines a bounded map $P_{F}^{(p)}: L_{p}(\mathcal{M}) \rightarrow L_{p}(\mathcal{M})$ given by $P_{F}^{(p)}: D^{\frac{1}{2 p}} x D^{\frac{1}{2 p}} \mapsto$ $D^{\frac{1}{2 p}} P_{F}(x) D^{\frac{1}{2 p}}$.

Proof. Let $x=\sum_{i} c_{i} W\left(e_{i}\right) \in \mathcal{A}$. As for any $i \in F$ its length is bounded by $n$ we have that $\left\|W\left(e_{i}\right)^{*} W\left(e_{i}\right)\right\| \leq C$ for some constant $C$. We get that $\sum_{i \in F}\left|c_{i}\right|^{2}\left\|W\left(e_{i}\right)^{*} W\left(e_{i}\right)\right\| \leq C \sum_{i \in F}\left|c_{i}\right|^{2}=$ $\|x\|_{2}^{2}$. Proposition 5.8 shows therefore that we get the first inequality in

$$
\left\|P_{F}(x)\right\|_{\text {bmo }} \leq \sqrt{2}\left\|P_{F}(x)\right\|_{2} \leq \sqrt{2}\|x\|_{2} \leq \sqrt{2}\|x\|_{\infty} .
$$

In Section 5.3 we show that $\left(\Psi_{t}\right)_{t \geq 0}$ has a Markov dilation with a.u. continuous path. We then get $L_{p} \rightarrow L_{p}$ boundedness of $P_{F}$ by interpolation, see Theorem 3.15 .

5.3. A Markov dilation for the radial semi-group of free Araki-Woods factors. In this Section we show that the radial semi-group on free Araki-Woods factors has a good reversed Markov dilation. The first step in the proof of Proposition 5.10 is due to Ricard (see the final remarks of [Ric08]). We need to find a suitable analogue for semi-groups which we do by an ultraproduct argument. Similar techniques were used in [Arh16], [Arh17] though in this case through quantization we can give a shorter argument directly on the Hilbert space level, see also the comment below this proposition.

Proposition 5.10. For $t \geq 0$ consider the Markov semi-group $\Psi_{t}=\Phi_{e^{-t}}$ where $\Phi_{r}, 0<r \leq 1$ is the Markov map on $\mathcal{M}$ determined by (5.14). $\Psi_{t}$ admits a $\varphi_{\Omega}$-modular Markov dilation with a.u. continuous path.

Proof. For $t \geq 0$. Set $T_{t} \in B(\mathcal{H})$ by $T_{t} \xi=e^{-t} \xi$. The proof splits in steps.

Step 1: Constructing a dilation for subsemi-groups of $\left(T_{t}\right)_{t \geq 0}$. Firstly for each $t \geq 0$ we may find a Hilbert space $\mathcal{K}$ containing $\mathcal{H}$ with orthogonal projection $P_{\mathcal{H}}: \mathcal{K}_{t} \rightarrow \mathcal{H}$ and a unitary $U_{t} \in B(\mathcal{K})$ such that for every $l \in \mathbb{N}$,

$$
\left.P_{\mathcal{H}} U_{t}^{l}\right|_{\mathcal{H}}=T_{t}^{l}=T_{t l} .
$$


Indeed, the Hilbert space $\mathcal{K}=\ell_{2}(\mathbb{Z}) \otimes \mathcal{H}$ with unitary $U_{t}, t=-\log (r)$ acting on the first tensor leg by

$$
\left(\begin{array}{ccccccccccccc}
\ddots & \vdots & \vdots & & & & & & & & & & \\
\ldots & 1 & 0 & 0 & \ldots & & & & & & & & \\
\ldots & 0 & 1 & 0 & \ldots & & & & & & & & \\
\ldots & 0 & 0 & 1 & \ldots & & & & & & & & \\
& \vdots & \vdots & \vdots & \ddots & & & & & & & & \\
& & & \ldots & 1 & 0 & 0 & 0 & \ldots & & & \\
& & \ldots & 0 & \sqrt{1-r^{2}} & r & 0 & \ldots & & & \\
& & & \ldots & 0 & r & \sqrt{1-r^{2}} & 0 & \ldots & & & \\
& & \ldots & 0 & 0 & 0 & 1 & \ldots & & & & \\
& & & & & & & & \ddots & \vdots & \vdots & & \\
& & & & & & & & \ldots & 1 & 0 & 0 & \ldots \\
& & & & & & & & \ldots & 0 & 1 & 0 & \ldots \\
& & & & & & & & \ldots & 0 & 0 & 1 & \ldots \\
& & & & & & & & & \vdots & \vdots & \vdots & \ddots
\end{array}\right),
$$

where the bottom left entry $r$ is located at position $(0,0)$. So $U_{t}$ acts as a shift operator on $\ell_{2}(\mathbb{Z} \backslash\{0,1\}) \otimes \mathcal{H}$. $\mathcal{H}$ is a subspace of $\mathcal{K}$ by the embedding

$$
J: \xi \mapsto \delta_{0} \otimes \xi \in \ell_{2}(\mathbb{Z}) \otimes \mathcal{H}=\ell_{2}(\mathbb{Z}, \mathcal{H}) .
$$

(5.16) is then elementary to check (see also [Pis96, Theorem 1.1]). We let $P_{t, n}$ be the orthogonal projection onto the closed linear span of $\left\{U_{t}^{l} \xi \mid l \geq n, \xi \in \mathcal{H}\right\}$. We get that for $\xi, \eta \in \mathcal{H}$ we have for $l, n \geq 0$

$$
\left\langle\xi, U_{t}^{n+l} \eta\right\rangle=\left\langle\xi, P_{\mathcal{H}} U_{t}^{n+l} P_{\mathcal{H}} \eta\right\rangle=\left\langle T_{t}^{n+l} \xi, \eta\right\rangle=\left\langle T_{t}^{n} \xi, U_{t}^{l} \eta\right\rangle=\left\langle U_{t}^{n} T_{t}^{n} \xi, U_{t}^{n+l} \eta\right\rangle .
$$

Which shows that $P_{t, n} P_{\mathcal{H}} \xi=U_{t}^{n} T_{t}^{n} \xi$. Moreover from (5.17) we get for $n \geq k$ that $\left\langle U_{t}^{k} \xi, U_{t}^{n+l} \eta\right\rangle=$ $\left\langle U_{t}^{n} T_{t}^{n-k} \xi, U_{t}^{n+l} \eta\right\rangle$. So we find that $P_{t, n} U_{t}^{k} \xi=U_{t}^{n} T_{t}^{n-k} \xi$. So if we put $J_{t, n}=U_{t}^{n} J: \mathcal{H} \rightarrow \mathcal{K}$ we get that

$$
P_{t, n} J_{t, k}=J_{t, n} T_{t}^{n-k}, \quad n \leq k .
$$

This is a discrete Hilbert space version of the reversed Markov dilation property (2.5).

Step 2: Constructing a Markov dilation. We shall now construct a continuous version of (5.18), To do so, for $t \geq 0$ let $\mathcal{K}_{t}:=\mathcal{K}$ be the Hilbert space as in the previous paragraph and let $J_{t, n}: \mathcal{H} \rightarrow \mathcal{K}_{t}$ be the injection as before. Also let $P_{t, n}$ and $U_{t}$ be as before.

Set groups $G_{m}=2^{-m} \mathbb{Z}$ and $G=\cup_{m \geq 1} G_{m}$. The group $G$ is understood as a topological group with the Euclidian topology inherited from $\mathbb{R}$. Let $\mathcal{U}$ be a non-principal ultrafilter on $\mathbb{N}$. Consider $\mathcal{K}_{\mathcal{U}}=\prod_{m, \mathcal{U}} \mathcal{K}_{2^{-m}}$. Let $K: \mathcal{H} \rightarrow \mathcal{K}_{\mathcal{U}}$ be the embedding sending $\xi$ to the constant family $(\xi)_{\mathcal{U}}$. Let $P_{\mathcal{H}}=K^{*}$ be the projection onto $\mathcal{H}$. For $t \in \mathrm{G}$ we define the unitary $V_{t, m}$ on $\mathcal{K}_{2^{-m}}$ by

$$
V_{t, m}= \begin{cases}U_{2^{-m}}^{2^{m}} & \text { if } t \in \mathrm{G}_{m}, \\ \operatorname{Id}_{\mathcal{K}_{2-m}} & \text { otherwise. }\end{cases}
$$

Then for $t \in \mathrm{G}$ set $V_{t}=\left(V_{t, m}\right) \mathcal{U}$ which is a unitary on $\mathcal{K}_{\mathcal{U}}$. We claim that the assignment

$$
\mathrm{G} \ni t \mapsto V_{t} P_{\mathcal{K}}
$$


is strong-* continuous. Indeed, let $\xi \in \mathcal{H}$ be a unit vector. Let $t, s \in \mathrm{G}$ and assume that $s \geq t \geq 0$. Then let $M$ be such that for any $m>M$ we have $s, t \in \mathrm{G}_{m}$. Fix such $m>M$. We get that

$$
\begin{aligned}
U_{2^{-m}}^{t 2^{m}} \xi= & \left(0, \ldots, 0, \sqrt{1-r^{2}} \xi, \sqrt{1-r^{2}} r \xi, \ldots,\right. \\
& \left.\ldots, \sqrt{1-r^{2}} r^{t 2^{m}-3} \xi, \sqrt{1-r^{2}} r^{t 2^{m}-2} \xi, \sqrt{1-r^{2}} r^{t 2^{m}-1} \xi, r^{t 2^{m}} \xi, 0,0, \ldots\right) .
\end{aligned}
$$

Recalling $r=e^{-2^{-m}}$ this shows that we get from a small elementary computation,

$$
\begin{aligned}
& \left\|\left(U_{2^{-m}}^{s 2^{m}}-U_{2^{-m}}^{t 2^{m}}\right) \xi\right\|_{2}^{2} \\
= & \left(r^{s 2^{m}}-r^{t 2^{m}}\right)^{2}+\left(1-r^{2}\right) \sum_{l=1}^{t 2^{m}}\left(r^{s 2^{m}-l}-r^{t 2^{m}-l}\right)^{2}+\left(1-r^{2}\right) \sum_{l=t 2^{m}+1}^{s 2^{m}}\left(r^{s 2^{m}-l}\right)^{2} \\
= & \left(e^{-2 s}-e^{-2 t}\right)^{2}+\left(e^{-2 s}-e^{-2 r}\right)^{2}\left(e^{-2 t}-1\right)+e^{-2(s-t)}\left(e^{-2(s-t)}-1\right),
\end{aligned}
$$

which converges to 0 as $s \rightarrow t$. This shows that the unitary group $t \mapsto V_{t} P_{\mathcal{H}}$ is strong-* continuous. We extend (5.19) to a strongly continuous map $\mathbb{R} \ni t \rightarrow V_{t} P_{\mathcal{H}}$. This shows that we get an isometric embedding for every $t \in \mathbb{R}$,

$$
K_{t}: \mathcal{H} \rightarrow \mathcal{K}_{\mathcal{U}}: \xi \mapsto V_{t} K \xi
$$

For $t \in \mathrm{G}$ and $m \in \mathbb{N}_{\geq 1}$ we define

$$
Q_{t, m}= \begin{cases}P_{2^{-m}, s 2^{m}} & \text { if } t \in \mathrm{G}_{m} \\ 0 & \text { otherwise. }\end{cases}
$$

Then set $Q_{t}=\left(Q_{t, m}\right)_{m, \mathcal{U}}$. We claim that the mapping $\mathrm{G} \ni t \mapsto Q_{t}$ is decreasing and strongly continuous. Indeed we have for $t \in \mathrm{G}_{m}$ that $P_{2^{-m}, t 2^{m}}=P_{2^{-m}, 0} U_{2^{-m}}^{-t 2^{m}}$. Set $P=\left(P_{2^{-m}, 0}\right)_{\omega}$. So that for $t \in \mathrm{G}$ we have $Q_{t}=P V_{t}^{*}$. A computation similar to (2.2) shows that the function $\mathrm{G} \ni t \mapsto P V_{t}^{*}$ is weakly continuous. But as $Q_{t}$ is decreasing this convergence actually holds in the strong topology (see [Mur90, Theorem 4.1.1]) and by self-adjointness in the strong-*-topology. Therefore we obtain a decreasing strong-* continuous map $\mathbb{R} \ni t \mapsto Q_{t}$.

For $s, t \in \mathrm{G}, s \geq t$ and any $m$ large such that $s, t \in \mathrm{G}_{m}$. We get that for $\xi \in \mathcal{H}$,

$$
Q_{s, m} V_{t, m} \xi=P_{2^{-m}, s 2^{m}} U_{2^{-m}}^{t 2^{m}} \xi=U_{2^{-m}}^{s 2^{m}} T_{2^{-m}}^{(s-t) 2^{m}} \xi=V_{s, m} T_{s-t} \xi
$$

This shows that for $s, t \in \mathrm{G}, s \geq t$ we get that $Q_{s} V_{t} J=V_{s} J T_{s-t}$. By strong continuity we get $Q_{s} V_{t} J=V_{s} J T_{s-t}$ for all $s \geq t \geq 0$. So by definition

$$
Q_{s} J_{t}=J_{s} T_{s-t} \quad \text { for all } s \geq t \geq 0 .
$$

We finish the proof by quantization. Let $\left(V_{t}^{\mathcal{K}}\right)_{t \in \mathbb{R}}=\left(\operatorname{Id}_{B\left(\ell_{2}(\mathbb{Z})\right)} \otimes V_{t}\right)_{t \in \mathbb{R}}$ be the orthogonal transformation group on $\mathcal{K}_{\mathbb{R}}=\ell_{2}(\mathbb{Z}) \otimes \mathcal{H}_{\mathbb{R}}$. We set $\mathcal{N}=\Gamma\left(\mathcal{K},\left(V_{t}^{\mathcal{K}}\right)_{t \in \mathbb{R}}\right)$ and $\mathcal{N}_{s}=\Gamma\left(Q_{s} \mathcal{K}\right), s \geq 0$. By second quantization we get a conditional expectation $\mathcal{E}_{s}:=\Gamma\left(Q_{s}\right): \mathcal{N} \rightarrow \mathcal{N}_{s}$ and a normal injective $*$-homomorphism $\pi_{s}=\Gamma\left(J_{s}\right)$. By (5.21) they satisfy

$$
\mathcal{E}_{s} \circ \pi_{t}=\pi_{s} \circ \Psi_{s-t}, \quad 0 \leq t \leq s .
$$

It is clear from Remark 5.3 that this dilation is modular.

Step 3: A.u. continuity. Suppose that $\xi_{i}$ is a net of vectors in $\mathcal{H}$ converging in norm to $\xi \in \mathcal{H}$. Then we have for creation operators $a\left(\xi_{t}\right) \rightarrow a(\xi)$ in norm. By the Wick Theorem 5.1 we see that $W\left(\xi_{t}\right) \rightarrow W(\xi)$ in norm. Now for $x=W(\xi) \in \mathcal{A}, \xi \in \mathcal{H}$ the martingale $m_{t}(x)=\mathcal{E}_{t}\left(\pi_{t}(x)\right)=$ $W\left(Q_{t} J_{t} \xi\right)$ is norm continuous. 
Remark 5.11. Proposition 5.10 could potentially also be derived from a suitable analogue of [NFBK10, Theorem 7.1], provided that in this theorem one can keep track of the location of a specified real Hilbert subspace.

Acknowledgements. The author thanks A. González-Perez and M. Junge for useful disucssions on BMO-multipliers. The author thanks M. Veraar for pointing out [NFBK10] and Remark 5.11. The author thanks the referee for useful remarks leading to an improvement of the manuscript.

\section{REFERENCES}

[Arh16] C. Arhancet, Dilations of semigroups on von Neumann algebras and noncommutative $L_{p}$-spaces, arXiv:1603.04901.

[Arh17] C. Arhancet, S. Fackler, C. Le Merdy, Isometric dilations and $H^{\infty}$ calculus for bounded analytic semigroups and Ritt operators, Trans. Amer. Math. Soc. 369 (2017), no. 10, 6899-6933.

[Ana06] C. Anantharaman-Delaroche, On ergodic theorems for free group actions on noncommutative spaces, Probab. Theory Related Fields 135 (4) (2006), 520-546.

[Avs11] S. Avsec, Strong Solidity of the q-Gaussian Algebras for all $-1<q<1$, arXiv: 1110.4918.

[BeLö76] J. Bergh, J. Löfström, Interpolation spaces. An introduction. Grundlehren der Mathematischen Wissenschaften, No. 223. Springer-Verlag, Berlin-New York, 1976. x+207 pp.

[BKS07] M. Bozejko, B. Kümmerer, R. Speicher, q-Gaussian processes: noncommutative and classical aspects, Comm. Math. Phys. 185 (1997), 129-154.

[BrOz08] N. Brown, N. Ozawa, $C^{*}$-algebras and finite-dimensional approximations, Graduate Studies in Mathematics, 88. American Mathematical Society, Providence, RI, 2008. xvi+509 pp.

[Cad17] L. Cadilhac, Weak boundedness of Calderón-Zygmund operators on noncommutative $L^{1}$-spaces, arXiv: 1702.06536.

[Cas13] M. Caspers, The $L^{p}$-Fourier transform on locally compact quantum groups, J. Operator Theory 69 (2013), 161-193.

[CaSk15] M. Caspers, A. Skalski, The Haagerup approximation property for von Neumann algebras via quantum Markov semigroups and Dirichlet forms, Comm. Math. Phys. 336 (2015), 1637-1664.

[CPPR15] M. Caspers, J. Parcet, M. Perrin, R. Ricard, Noncommutative de Leeuw theorems, Forum Math. Sigma 3 (2015), e21, 59 pp.

[CXY13] Z. Chen, Q. Xu, Z. Yin, Harmonic analysis on quantum tori, Comm. Math. Phys. 322 (2013), no. 3, 755-805.

[CFK14] F. Cipriani, U. Franz, A. Kula, Symmetries of Lévy processes on compact quantum groups, their Markov semigroups and potential theory, J. Funct. Anal. 266 (2014), no. 5, 2789-2844.

[CiSa03] F. Cipriani, J.L. Sauvageot, Derivations as square roots of Dirichlet forms, J. Funct. Anal. 201 (2003), no. $1,78-120$.

[Con80] A. Connes, On the spatial theory of von Neumann algebras, J. Funct. Anal. 35 (1980), 153-164.

[Con73] A. Connes, Une classification des facteurs de type III, Ann. Sci. cole Norm. Sup. (4) 6 (1973), $133-252$.

[CoHa89] M. Cowling, U. Haagerup, Completely bounded multipliers of the Fourier algebra of a simple Lie group of real rank one, Invent. Math. 96 (1989), no. 3, 507-549.

[EfRu00] E. Effros, Z.-J. Ruan, Operator spaces, London Mathematical Society Monographs. New Series, 23. The Clarendon Press, Oxford University Press, New York, 2000. xvi+363 pp.

[Eym64] P. Eymard, L'algèbre de Fourier d'un groupe localement compact, Bull. Soc. Math. France 92 1964 $181-236$.

[FeSt72] C. Fefferman, E.M. Stein, $H^{p}$ spaces of several variables, Acta Math. 129 (1972), no. 3-4, $137-193$.

[GJP17a] A. M. González-Pérez, M. Junge, J. Parcet, Smooth Fourier multipliers in group algebras via Sobolev dimension, Ann. Sci. cole Norm. Sup. (to appear).

[GJP17b] A.M. González-Pérez, M. Junge, J. Parcet, Singular integrals in quantum Euclidean spaces, arXiv: 1705.01081.

[Gol84] S. Goldstein, Conditional expectations in $L_{p}$-spaces over von Neumann algebras, In: Quantum probability and applications, II (Heidelberg, 1984), Lecture Notes in Math., 1136, Springer, Berlin, 1985, 233-239.

[Gra08] L. Grafakos, Classical Fourier analysis, Second edition. Graduate Texts in Mathematics, 249. Springer, New York, 2008.

[Gra09] L. Grafakos, Modern Fourier analysis, Second edition. Graduate Texts in Mathematics, 250. Springer, New York, 2009. 
[Haa77] U. Haagerup, $L^{p}$-spaces associated with an arbitrary von Neumann algebra, Algèbres d'opérateurs et leurs applications en physique mathématique, Proc. Colloq., Marseille 1977, 175-184.

[HJX10] U. Haagerup, M. Junge, Q. Xu, A reduction method for noncommutative $L_{p}$-spaces and applications, Trans. Amer. Math. Soc. 362 (2010), no. 4, 2125-2165.

[Hia01] F. Hiai, q-deformed Araki-Woods algebras, Operator algebras and mathematical physics (Constanta, 2001), 169-202. Theta, Bucharest, 2003.

[Hil81] M. Hilsum, Les espaces $L^{p}$ d'une algèbre de von Neumann définies par la derivée spatiale, J. Funct. Anal. 40 (1981), 151-169.

[HoRi11] C. Houdayer, E. Ricard, Approximation properties and absence of Cartan subalgebra for free Araki-Woods factors, Adv. Math. 228 (2011), no. 2, 764-802.

[Izu97] H. Izumi, Constructions of non-commutative $L^{p}$-spaces with a complex parameter arising from modular actions, Internat. J. Math. 8 (1997), no. 8, 1029-1066.

[JoMa04] P. Jolissaint, F. Martin, Algèbres de von Neumann finies ayant la propriété de Haagerup et semigroupes $L^{2}$-compacts, Bull. Belg. Math. Soc. Simon Stevin 11 (1), 35-48 (2004).

[Jun02] M. Junge, Doob's inequality for non-commutative martingales, J. Reine Angew. Math. 549 (2002), $149-190$.

[JuSh05] M. Junge, D. Sherman, Noncommutative $L_{p}$ modules, J. Operator Theory 53 (2005), no. 1, 3-34.

[JuXu07] M. Junge, Q. Xu, Noncommutative maximal ergodic theorems, J. Amer. Math. Soc. 20 (2007), no. 2, 385-439.

[JuMe10] M. Junge, T. Mei, Noncommutative Riesz transformsa probabilistic approach, Amer. J. Math. 132 (2010), no. 3,611680 .

[JuMe12] M. Junge, T. Mei, BMO spaces associated with semigroups of operators, Math. Ann. 352 (2012), no. 3, 691-743.

[JMP14] M. Junge, T. Mei, J. Parcet, Smooth Fourier multipliers on group von Neumann algebras, Geom. Funct. Anal. 24 (2014), no. 6, 1913-1980.

[JuPe14] M. Junge, M. Perrin, Mathilde Theory of $H^{p}$-spaces for continuous filtrations in von Neumann algebras, Astrisque No. 362 (2014), vi+134 pp.

[JRS] M. Junge, E. Ricard, D. Shlyakhtenko, Paper in preparation.

[Mei17] T. Mei, BMO estimate of lacunary Fourier series on nonabelian discrete groups, arXiv:1703.02208.

[MeRi16] T. Mei, E. Ricard, Free Hilbert Transforms, to appear in Duke Math. J., arXiv:1605.02125.

[Mur90] G. Murphy, C $C^{*}$-algebras and operator theory, Academic Press, Inc., Boston, MA, 1990. x+286 pp.

[NFBK10] B. Sz.-Nagy, C. Foias, H. Bercovici, L. Kérchy, Harmonic analysis of operators on Hilbert space, Second edition. Revised and enlarged edition. Universitext. Springer, New York, 2010. xiv+474 pp.

[OkTo15] R. Okayasu, R. Tomatsu, Haagerup approximation property for arbitrary von Neumann algebras, Publ. Res. Inst. Math. Sci. 51 (2015), no. 3, 567-603.

[OzPo10] N. Ozawa, S. Popa, On a class of II 1 factors with at most one Cartan subalgebra II, Amer. J. Math. 132 (2010), no. 3, 841-866.

[Par09] J. Parcet, Pseudo-localization of singular integrals and noncommutative Calderón-Zygmund theory, J. Funct. Anal. 256 (2009), no. 2, 509-593.

[Pet09] J. Peterson, L2-rigidity in von Neumann algebras, Invent. Math. 175 (2009), no. 2, 417-433.

[Pis96] G. Pisier, Similarity problems and completely bounded maps, Lecture Notes in Mathematics, 1618. SpringerVerlag, Berlin, 1996. viii+156 pp.

[Pis02] G. Pisier, Introduction to operator space theory, London Mathematical Society Lecture Note Series, 294. Cambridge University Press, Cambridge, 2003.

[Ric08] E. Ricard, A Markov dilation for self-adjoint Schur multipliers, Proc. Amer. Math. Soc. 136 (2008), no. 12, 4365-4372.

[Ric16] E. Ricard, $L_{p}$-multipliers on quantum tori, J. Funct. Anal. 270 (2016), no. 12, 4604-4613.

[Sau99] J.L. Sauvageot, Strong Feller semigroups on $C^{*}$-algebras, J. Operator Theory 42 (1999), no. 1, 83102.

[Shl97] D. Shlyakhtenko, Free quasi-free states, Pacific J. Math. 177 (1997), 329-368.

[StVa74] S. Stroock, D. Varadhan, A probabilistic approach to $H_{p}\left(\mathbb{R}^{d}\right)$, Trans. Am. Math. Soc. 192, 245-260 (1974).

[Tak02] M. Takesaki, Theory of operator algebras. I. Encyclopaedia of Mathematical Sciences, 124. Operator Algebras and Non-commutative Geometry, 5. Springer-Verlag, Berlin, 2002. xx+415 pp.

[Tak03] M. Takesaki, Theory of operator algebras. II. Encyclopaedia of Mathematical Sciences, 125. Operator Algebras and Non-commutative Geometry, 6. Springer-Verlag, Berlin, 2003.

[Ter81] M. Terp, $L^{p}$ spaces associated with von Neumann algebras, Notes, Report No. 3a $+3 \mathrm{~b}$, Københavns Universitets Matematiske Institut, Juni 1981. 
[Ter82] M. Terp, Interpolation spaces between a von Neumann algebra and its predual, J. Operator Theory 8 (1982), 327-360.

[Var85] N. Th. Varopoulos, Hardy-Littlewood theory for semigroups, J. Funct. Anal. 63 (2), 240-260 (1985).

[Was17] M. Wasilewski, q-Araki-Woods algebras: extension of second quantisation and Haagerup approximation property, Proc. Amer. Math. Soc. 145 (2017), 5287-5298.

[XXX16] R. Xia, X. Xiong, Q. Xu, Characterizations of operator-valued Hardy spaces and applications to harmonic analysis on quantum tori, Adv. Math. 291 (2016), 183-227.

[XuYa05a] T.D. Xuan, L. Yan, Duality of Hardy and BMO-spaces associated with operators with heat kernel bounds, J. Amer. Math. Soc. 18 (2005), no. 4, 943-973.

[XuYa05b] T.D. Xuan, L. Yan, New function spaces of bmo type, the John-Nirenberg inequality, interpolation, and applications, Commun. Pure Appl. Math. 58 (10), 1375-1420 (2005).

TU Delft, EWI/DiAM, P.O.Box 5031, 2600 GA Delft, The Netherlands

E-mail address: m.p.t.caspers@tudelft.nl 